\title{
Chiral study of the $a_{0}(980)$ resonance and $\pi \eta$ scattering phase shifts in light of a recent lattice simulation
}

\author{
Zhi-Hui Guo ${ }^{a, b}$, Liuming Liu ${ }^{b}$, Ulf-G. Meißner ${ }^{b, c}$, J. A. Oller ${ }^{d}, \quad$ A. Rusetsky ${ }^{b}$ \\ ${ }^{a}$ Department of Physics, Hebei Normal University, Shijiazhuang 050024, China \\ ${ }^{b}$ Helmholtz-Institut für Strahlen- und Kernphysik and Bethe Center for Theoretical Physics, \\ Universität Bonn, D-53115 Bonn, Germany \\ ${ }^{c}$ Institute for Advanced Simulation, Institut für Kernphysik and Jülich Center for Hadron Physics, \\ Forschungszentrum Jülich, D-52425 Jülich, Germany \\ ${ }^{d}$ Departamento de Física. Universidad de Murcia. E-30071 Murcia. Spain
}

\begin{abstract}
We investigate the $a_{0}(980)$ resonance within chiral effective field theory through a threecoupled-channel analysis, namely $\pi \eta, K \bar{K}$ and $\pi \eta^{\prime}$. A global fit to recent lattice finitevolume energy levels from $\pi \eta$ scattering and relevant experimental data on a $\pi \eta$ event distribution and the $\gamma \gamma \rightarrow \pi \eta$ cross section is performed. Both the leading and next-toleading-order analyses lead to similar and successful descriptions of the finite-volume energy levels and the experimental data. However, these two different analyses yield different $\pi \eta$ scattering phase shifts for the physical masses for the $\pi, K, \eta$ and $\eta^{\prime}$ mesons. The inelasticities, the pole positions in the complex energy plane and their residues are calculated both for unphysical and physical meson masses.
\end{abstract}

PACS numbers: 12.39.Fe, 13.75.Lb, 12.38.Gc

Key words: Chiral Lagrangian, Meson-meson interaction, Lattice field theory

\section{Introduction}

The nonperturbative meson-meson dynamics of low-energy QCD, especially in the scalar channels, is one of the most challenging research topics in hadron physics. The complexity of the strong meson-meson interactions is manifested in many resonances that appear in various scattering processes [1]. Well-known examples are the $f_{0}(500)$ (or $\sigma$ ) in $\pi \pi$ scattering, the $f_{0}(980)$ in $\pi \pi$ and $K \bar{K}$ coupled channels, the $a_{0}(980)$ in $\pi \eta$ and $K \bar{K}$ scattering, and the $K_{0}^{*}(800)$ (or $\left.\kappa\right)$ in the $\pi K$ channel. Though it seems plausible that the light isoscalars $f_{0}(500)$ and $f_{0}(980)$, the isovector $a_{0}(980)$ and the isospin one-half $K_{0}^{*}(800)$ may form a nonet [2], the situation for those scalars is much less clear than for the vector nonet $\rho(770), K^{*}(892), \omega(778)$ and $\phi(1020)$.

A reliable way to obtain further insights into these scalar mesons is based on the lowenergy effective field theory of QCD, chiral perturbation theory $(\chi \mathrm{PT})$, and the unitarity and 
analyticity requirements of the corresponding scattering amplitudes [3]. In this approach, one usually needs scattering data, such as the phase shifts or inelasticities, as inputs to constrain the free parameters. In the last decade, enormous progress has been made in the study of the $f_{0}(500)$, see Ref. [8] for a recent review. It is most likely that the effects from the inelastic channels, such as $K \bar{K}$ and other higher ones, are small for the $f_{0}(500)$. As a result, one can use the single-channel formalism to describe this broad resonance well. In addition, many existing precise $\pi \pi$ scattering data also help to precisely determine the $f_{0}(500)$ pole position. The experimental $\pi K$ phase shifts also confirm the existence of the $K_{0}^{*}(800)$ as a pole in the complex energy plane [5-7, 11]. Due to the proximity of the $f_{0}(980)$ and $a_{0}(980)$ to the $K \bar{K}$ threshold, the coupled-channel formalism is essential to study these two states. Rigorous dispersive studies have been performed for the $f_{0}(980)$, see Ref. [12] and references therein. Various unitarized $\chi \mathrm{PT}$ approaches also confirm that there is a well-established resonance pole for the $f_{0}(980)$ after successfully reproducing the $\pi \pi$ scattering data around $1 \mathrm{GeV}$ [4, 6, 7, 13]. However, the situation for the $a_{0}(980)$ is less clear and its pole positions are still under debate [6, 7, 14, 17]. One of the biggest difficulties in preventing a precise determination of the $a_{0}(980)$ is the lack of direct experimental $\pi \eta$ scattering data. It is unlikely that this will be improved in the near future.

Fortunately, important progress using lattice QCD simulations for $\pi \eta$ scattering, together with $K \bar{K}$ and $\pi \eta^{\prime}$ coupled channels, has been made very recently [17. However, the pion mass $\left(m_{\pi} \sim 391 \mathrm{MeV}\right)$ used in the calculation is still much heavier than its physical value. A large number of energy levels in the finite volume is obtained by using a large amount of interpolating operators and many moving frames. These energy levels are then used to extract the $\pi \eta$ phase shifts and inelasticities by using Lüscher's method [18] and parametrizing the $K$ matrix in various ways. The resulting $\pi \eta$ phase shifts [17] around the $K \bar{K}$ threshold do not show any sharp increase, and hence they do not correspond to the behavior of a canonical resonance pole in the complex energy plane. Instead the authors of Ref. [17] find that the $a_{0}(980)$ state corresponds to a pole in the fourth Riemann sheet (RS), which is not directly connected to the physical sheet2. Although this observation is made with $m_{\pi}=391 \mathrm{MeV}$, interestingly it agrees with the previous study in Ref. [16] for the $a_{0}(980)$, which also corresponds to a fourth RS pole. But the calculation in Ref. [16] is done with the physical masses for the $\pi, K, \eta$ and $\eta^{\prime}$ channels.

In order to make a close comparison with the physical $a_{0}(980)$ state, a proper way to perform the chiral extrapolation of the lattice simulations in Ref [17] is essential. In this respect, the $\chi \mathrm{PT}$ framework provides a reliable tool. In this work we use the unitarized $\chi \mathrm{PT}$ approach [4,7, 23] to reanalyze the lattice simulations, and then extrapolate the $\pi, K$, $\eta$ and $\eta^{\prime}$ masses to their physical values. It is worth emphasizing that the methodology for coupled-channel unitarized $\chi \mathrm{PT}$ in a finite volume for the scalar meson sector was developed in Refs. [24 27]. Note also that, recently, a similar method was used in Ref. [28] in order to extract the position of the $\rho$-meson pole from the lattice phase shifts. In particular, it has been argued that the coupling to the $K \bar{K}$ channel might have a significant impact on it. Instead of analyzing the phase shifts provided in Ref [17, we directly fit the lattice energy

\footnotetext{
${ }^{1}$ Note that, recently, the Lüscher method has become a commonly accepted tool to analyze the lattice data in the scattering sector, including the case of the multi-channel scattering (see, e.g., Refs. [17, 19, 21]). Different algebraic parameterizations for the $K$-matrix are used and the free parameters are fitted to the lattice data on the energy levels. Note also that an alternative approach to study of the inelastic scattering has been formulated recently $[22$.

${ }^{2}$ The pole on the fourth RS in Ref. 17] lies above the $K \bar{K}$ threshold. The physical sheet in that energy region is directly connected to the third $\mathrm{RS}$, in which a canonical resonance pole should be located.
} 
levels by considering unitarized $\chi \mathrm{PT}$ in a finite box. In addition to the lattice finite-volume energy levels, we also include two kinds of experimental data in the global fits, namely, a $\pi \eta$ event distribution [29] and the $\gamma \gamma \rightarrow \pi \eta$ cross section [30], so as to better constrain the free parameters in the analyses. After the successful reproduction of the lattice energy levels and experimental data, we then calculate the $\pi \eta$ phase shifts, inelasticities, pole positions, and their residues by taking both heavy unphysical and physical masses for $\pi, K, \eta$ and $\eta^{\prime}$.

The article is organized as follows. We introduce the unitarized $\chi \mathrm{PT}$ approach and the finite-volume effects in moving frames in Sec. 2. The fits to the lattice energy levels and experimental data are analyzed in detail in Sec. 3. The $\pi \eta$ scattering phase shifts, inelasticities, the $a_{0}(980)$ and $a_{0}(1450)$ pole positions and their residues for the unphysical masses are given in Sec. 4. The results after extrapolating the $\pi, K, \eta$ and $\eta^{\prime}$ masses to their physical values are discussed in Sec. 5. A short summary and conclusions are given in Sec. 6.

\section{$2 \quad$ Unitarized $U(3) \chi \mathrm{PT}$ and its finite-volume effects}

In this section we briefly review the basic aspects of the formalism used to analyze lattice QCD energy levels and experimental data. Note that $\chi \mathrm{PT}$ is the effective field theory of low-energy QCD and it has been proven to be quite successful to describe the dynamics of the pseudo-Nambu-Goldstone bosons (pNGBs), including the $\pi, K$ and $\eta$ mesons [31. In the present work we study the $a_{0}(980)$ by including the scattering of three coupled channels, namely $\pi \eta, K \bar{K}$ and $\pi \eta^{\prime}$. In this case, $U(3) \chi \mathrm{PT}[32,33$ is the proper framework, instead of the conventional $S U(3) \chi \mathrm{PT}$ [31. This is because the singlet $\eta_{0}$ and the QCD $U(1)_{A}$ anomaly effect are explicitly included in $U(3) \chi \mathrm{PT}$, while in the $S U(3)$ case the heavy singlet $\eta_{0}$ is integrated out. The leading-order (LO) Lagrangian of $U(3) \chi \mathrm{PT}$ reads 34

$$
\mathcal{L}_{2}=\frac{F^{2}}{4}\left\langle u_{\mu} u^{\mu}\right\rangle+\frac{F^{2}}{4}\left\langle\chi_{+}\right\rangle+\frac{F^{2}}{3} M_{0}^{2} \ln ^{2} \operatorname{det} u,
$$

where $\langle\ldots\rangle$ denotes the trace in flavor space and the last term encodes the $U_{A}(1)$ anomaly effect that gives the singlet $\eta_{0}$ a large mass $M_{0}$ even in the chiral limit. The basic chiral operators are defined as

$$
\begin{aligned}
u_{\mu} & =i u^{+} D_{\mu} U u^{+}, \\
\chi_{+} & =u^{+} \chi u^{+}+u \chi^{+} u, \\
U & =u^{2}=\exp (i \sqrt{2} \Phi / F), \\
D_{\mu} U & =\partial_{\mu} U-i r_{\mu} U+i U l_{\mu}, \\
\chi & =2 B(s+i p),
\end{aligned}
$$

where $F$ denotes the weak decay constant of the pNGBs in the chiral limit, the parameter $B$ is related to the quark condensate through $\left\langle 0\left|\bar{q}^{i} q^{j}\right| 0\right\rangle=-F^{2} B \delta^{i j}$ at leading order, $r_{\mu}, l_{\mu}, s, p$ are external sources and the pNGBs are collected in the $3 \times 3$ matrix

$$
\Phi=\left(\begin{array}{ccc}
\frac{1}{\sqrt{2}} \pi^{0}+\frac{1}{\sqrt{6}} \eta_{8}+\frac{1}{\sqrt{3}} \eta_{0} & \pi^{+} & K^{+} \\
\pi^{-} & \frac{-1}{\sqrt{2}} \pi^{0}+\frac{1}{\sqrt{6}} \eta_{8}+\frac{1}{\sqrt{3}} \eta_{0} & K^{0} \\
K^{-} & \bar{K}^{0} & \frac{-2}{\sqrt{6}} \eta_{8}+\frac{1}{\sqrt{3}} \eta_{0}
\end{array}\right)
$$


The explicit chiral symmetry breaking is realized by taking the vacuum expectation values of the scalar source $s=\operatorname{diag}\left(m_{u}, m_{d}, m_{s}\right)$, with $m_{q}$ the light-quark masses. We work in the isospin symmetry limit $m_{u}=m_{d}$.

The physical $\eta$ and $\eta^{\prime}$ states result from the mixing of the octet $\eta_{8}$ and the singlet $\eta_{0}$. At leading order, it is enough to introduce one mixing angle $\theta$ to diagonalize the quadratic terms of $\eta_{0}$ and $\eta_{8}$

$$
\begin{aligned}
& \eta_{8}=c_{\theta} \bar{\eta}+s_{\theta} \bar{\eta}^{\prime}, \\
& \eta_{0}=-s_{\theta} \bar{\eta}+c_{\theta} \bar{\eta}^{\prime},
\end{aligned}
$$

with $c_{\theta}=\cos \theta$ and $s_{\theta}=\sin \theta$. Here, we use the notation $\bar{\eta}$ and $\bar{\eta}^{\prime}$ to denote the diagonalized fields of the Lagrangian Eq. (10) at leading order. When higher order contributions are included, $\bar{\eta}$ and $\bar{\eta}^{\prime}$ will get mixed again and we refer to Refs. [16, 35, 36] for further details on handling the higher order mixing effects. The LO mixing angle $\theta$ can be calculated in terms of the singlet $\eta_{0}$ mass $M_{0}$ in the chiral limit and the LO masses of the pion and the kaon [16, 35.

$$
\sin \theta=-\left(\sqrt{1+\frac{\left(3 M_{0}^{2}-2 \Delta^{2}+\sqrt{9 M_{0}^{4}-12 M_{0}^{2} \Delta^{2}+36 \Delta^{4}}\right)^{2}}{32 \Delta^{4}}}\right)^{-1},
$$

where $\Delta^{2}=\bar{m}_{K}^{2}-\bar{m}_{\pi}^{2}$, and $\bar{m}_{K}$ and $\bar{m}_{\pi}$ are the LO kaon and pion masses, respectively.

The higher order contributions in $\chi \mathrm{PT}$ include both the chiral loops and the higher order low-energy constants (LECs). In Ref. [16] the one-loop calculation of all two-body light-meson scattering amplitudes is carried out within $U(3) \chi \mathrm{PT}$. A systematical study of the $O\left(p^{4}\right)$ Lagrangian of $U(3) \chi \mathrm{PT}$ is given in Refs. 32, 33]. Another way to account for the effects from the higher order LECs is to include resonance exchanges in a chiral invariant way [37. The pioneering study of resonance exchanges in the chiral framework for the $\pi \pi$ and $\pi K$ scattering was given in Ref. [38]. A generalization to include the leading resonance exchanges in the chiral counting in all the meson-meson scattering channels is completed in Ref. [16]. For a more detailed account the reader is referred to Ref. [16] and references therein.

\subsection{Brief reminder of unitarized $U(3) \chi \mathrm{PT}$}

Since $\chi \mathrm{PT}$ is organized in a double expansion in momenta and light-quark masses, it can only be applied for low-energy processes involving the pNGBs. In the higher energy region, especially when the resonances appear, the perturbative $\chi \mathrm{PT}$ amplitudes start to severely violate the unitarity condition and one can not trust the $\chi \mathrm{PT}$ expressions anymore. The unitarization procedure, which restores the unitarity of the perturbative $\chi \mathrm{PT}$ amplitudes, provides a useful tool to extend the $\chi \mathrm{PT}$ domain to the resonance energy region. However, this is usually done at the expense of violating crossing symmetry, and such a unitarization procedure unavoidably introduces some model dependence from the chosen set of higher order effects that are resummed. In the single $\pi \pi$ channel case, unitarity and analyticity can be strictly implemented within a range of energies and different groups obtain quite compatible results for the $f_{0}(500)$ pole positions [8]. However, a rigorous solution for the coupled-channel scattering is typically not possible and usually different types of approximations are introduced. A convenient way to proceed is to treat the right-hand cut (or the unitarity cut) nonperturbatively, whereas the cross-channel effects are included in a perturbative fashion [4, 23, 39]. Indeed, this is the case in many unitarized $\chi \mathrm{PT}$ studies [4, 6, 7]. 
A unitarization of the perturbative meson-meson scattering amplitudes up to the nextto-leading order (NLO) calculated in the one-loop $U(3) \chi \mathrm{PT}$ plus tree-level resonance exchanges [16] is then performed using the formalism of Ref. [7]. The final expression for the meson-meson scattering amplitude $\mathcal{T}_{J}^{I}(s)$ reads

$$
\mathcal{T}_{J}^{I}(s)=\left[1+N_{J}^{I}(s) \cdot G(s)\right]^{-1} \cdot N_{J}^{I}(s),
$$

where $I$ and $J$ denote the isospin and angular momentum, respectively. This unitarization method corresponds to an algebraic approximation of the conventional $N / D$ method [7]. By construction, the function $G(s)$ in Eq. (6) incorporates the two-body right-hand cut and it is given by the standard two-point one-loop function

$$
G(s)=i \int \frac{\mathrm{d}^{4} q}{(2 \pi)^{4}} \frac{1}{\left(q^{2}-m_{1}^{2}+i \epsilon\right)\left[(P-q)^{2}-m_{2}^{2}+i \epsilon\right]}, \quad s \equiv P^{2},
$$

which can be calculated by a once-subtracted dispersion relation or in the dimensional regularization by replacing the divergence with a constant. The explicit expression of $G(s)$ reads 7$]$

$$
\begin{aligned}
G(s)^{\mathrm{DR}}= & \frac{1}{16 \pi^{2}}\left\{a(\mu)+\ln \frac{m_{1}^{2}}{\mu^{2}}+\frac{s-m_{1}^{2}+m_{2}^{2}}{2 s} \ln \frac{m_{2}^{2}}{m_{1}^{2}}\right. \\
& +\frac{\sigma(s)}{2 s}\left[\ln \left(\sigma(s)+s-m_{2}^{2}+m_{1}^{2}\right)-\ln \left(\sigma(s)-s+m_{2}^{2}-m_{1}^{2}\right)\right. \\
& \left.\left.+\ln \left(\sigma(s)+s+m_{2}^{2}-m_{1}^{2}\right)-\ln \left(\sigma(s)-s-m_{2}^{2}+m_{1}^{2}\right)\right]\right\},
\end{aligned}
$$

where the superscript DR denotes the use of the dimensional regularization in the expression of $G(s), m_{1}$ and $m_{2}$ are the masses of the two intermediate mesons in the scattering process, $a(\mu)$ is the subtraction constant and

$$
\sigma(s)=\sqrt{\lambda\left(s, m_{1}^{2}, m_{2}^{2}\right)},
$$

with $\lambda(a, b, c)=a^{2}+b^{2}+c^{2}-2 a b-2 b c-2 a c$ the Källén function. The function $G(s)$ itself is independent of the renormalization scale $\mu$, since the explicit $\mu$ dependence in the second term in Eq. (8) is compensated by the subtraction constant $a(\mu)$. We will fix $\mu=770 \mathrm{MeV}$ in our later discussion, indicating that the value of the subtraction constant obtained here refers to that scale. The subtraction constants could be reabsorbed gradually when higher orders are included in the function $N(s)$. As it is clear from Eq. (6), for an exact elimination of the subtraction constants, the inverse of the new function $\widetilde{N}(s)$ would be $\widetilde{N}_{J}^{I}(s)^{-1}=N_{J}^{I}(s)^{-1}+\delta G$, with $\delta G$ the diagonal matrix including only the subtraction constants.

In contrast, the $N_{J}^{I}(s)$ function is free of any two-body right-hand cut singularity 3 and only contains the crossed-channel cuts. Its explicit expression is given by [16]

$$
N_{J}^{I}(s)=T_{J}^{I}(s)^{(2)+\operatorname{Res}+\text { Loop }}+T_{J}^{I}(s)^{(2)} \cdot G(s) \cdot T_{J}^{I}(s)^{(2)},
$$

where $T_{J}^{I}(s)^{(2)+\text { Res+Loop }}$ are the partial-wave projected $U(3) \chi \mathrm{PT}$ amplitudes, and the superscripts "(2), Res" and "Loop" denote the LO amplitudes, resonance exchanges and loop contributions, respectively. The explicit calculations of these perturbative amplitudes are given in

\footnotetext{
${ }^{3}$ Except those from the channels with heavier thresholds.
} 
detail in Ref. [16] and we briefly recapitulate the main results here. The LO $S$-wave amplitudes in the isospin $I=1$ channel are

$$
\begin{aligned}
T_{J=0}^{I=1, \pi \eta \rightarrow \pi \eta}(s)^{(2)} & =\frac{\left(c_{\theta}-\sqrt{2} s_{\theta}\right)^{2} m_{\pi}^{2}}{3 F_{\pi}^{2}} \\
T_{J=0}^{I=1, \pi \eta \rightarrow K \bar{K}}(s)^{(2)} & =\frac{c_{\theta}\left(3 m_{\eta}^{2}+8 m_{K}^{2}+m_{\pi}^{2}-9 s\right)+2 \sqrt{2} s_{\theta}\left(2 m_{K}^{2}+m_{\pi}^{2}\right)}{6 \sqrt{6} F_{\pi}^{2}} \\
T_{J=0}^{I=1, \pi \eta \rightarrow \pi \eta^{\prime}}(s)^{(2)} & =\frac{\left(\sqrt{2} c_{\theta}^{2}-c_{\theta} s_{\theta}-\sqrt{2} s_{\theta}^{2}\right) m_{\pi}^{2}}{3 F_{\pi}^{2}} \\
T_{J=0}^{I=1, K \bar{K} \rightarrow K \bar{K}}(s)^{(2)} & =\frac{s}{4 F_{\pi}^{2}}, \\
T_{J=0}^{I=1, K \bar{K} \rightarrow \pi \eta^{\prime}}(s)^{(2)} & =\frac{s_{\theta}\left(3 m_{\eta^{\prime}}^{2}+8 m_{K}^{2}+m_{\pi}^{2}-9 s\right)-2 \sqrt{2} c_{\theta}\left(2 m_{K}^{2}+m_{\pi}^{2}\right)}{6 \sqrt{6} F_{\pi}^{2}} \\
T_{J=0}^{I=1, \pi \eta^{\prime} \rightarrow \pi \eta^{\prime}}(s)^{(2)} & =\frac{\left(\sqrt{2} c_{\theta}+s_{\theta}\right)^{2} m_{\pi}^{2}}{3 F_{\pi}^{2}},
\end{aligned}
$$

where $c_{\theta}$ and $s_{\theta}$ are defined in Eq. (44).

Concerning the resonance exchanges, we mention that in Ref. [16] one multiplet of bare octet scalar resonances is included at the Lagrangian level, which is mostly responsible for the excited physical scalar states of $f_{0}(1370), K_{0}^{*}(1430)$ and $a_{0}(1450)$. The bare singlet scalar introduced at the Lagrangian level is found to be important for the $f_{0}(980)$. The other scalar resonances, such as $\sigma, \kappa$ and $a_{0}(980)$, are mainly generated from the nonperturbative meson-meson contact interactions. Concerning other higher order effects, such as the vector resonance exchanges and light pseudoscalar loop contributions, we refer to Ref. [16] for further details. The unknown parameters in our model, including the resonance couplings and the subtraction constants, were determined in Ref. [16] by fitting a large amount of experimental data, consisting of the $\pi \pi \rightarrow \pi \pi, K \bar{K}$ scattering phase shifts and inelasticities in the $I J=00$ channel [40], the $\pi \pi \rightarrow \pi \pi$ phase shifts with $I J=11$ [41] and $I J=20$ [42], the $\pi K \rightarrow \pi K$ phase shifts with $I J=\frac{1}{2} 0, \frac{3}{2} 0$ and $\frac{1}{2} 1$ [43] and a $\pi \eta$ event distribution in the $I J=10$ case [29]. Note that there is no available direct experimental data for $\pi \eta$ scattering, but the $I J=10$ partial-wave amplitudes can be tested because of their impact through final-state interactions. The $\pi \eta$ event distribution taken corresponds to the measured one in the complicated reaction $p p \rightarrow p p \eta \pi^{+} \pi^{-}$ [29. Since it is quite possible that the $K \bar{K}$ and $\pi \eta$ intermediate states may enter in different ways in $p p \rightarrow p p \eta \pi^{+} \pi^{-}$, we introduce two parameters $c_{1}$ and $c_{2}$ to account for the underlying mechanisms. By assuming that the energy dependence is dominated by the resonating finalstate interactions, we can then write the $\pi \eta$ event distribution near the $K \bar{K}$ threshold as [23,44]

$$
\frac{d N_{\pi \eta}}{d E_{\pi \eta}}=q_{\pi \eta}\left|c_{1} D^{-1}(s)_{\pi \eta \rightarrow \pi \eta}+c_{2} D^{-1}(s)_{\pi \eta \rightarrow K \bar{K}}\right|^{2},
$$

where $q_{\pi \eta}$ denotes the three-momentum of the $\pi \eta$ system in the center-of-mass (CM) frame, $E_{\pi \eta}=\sqrt{s}$ is the CM energy and the matrix function $D^{-1}(s)$ is defined as 23 .

$$
D^{-1}(s)=\left[1+N_{J}^{I}(s) \cdot G(s)\right]^{-1},
$$

such that the unitarized $T$ matrix, cf. Eq. (6) , can be written as $\mathcal{T}=D^{-1} \cdot N$. In general, the parameters $c_{1}$ and $c_{2}$ in Eq. (12) can be complex, but due to the irrelevance of an overall phase 
in the linear combination of Eq. (12), just one of the two parameters needs to be complex. For definiteness, we take $c_{2}$ to be real in later numerical discussions and treat $c_{1}$ as complex, if necessary. Note that in Refs. [16, 45] it is found that two real parameters are enough to reproduce the event distribution 4 .

In addition, we also include the experimental $\gamma \gamma \rightarrow \pi \eta$ cross section from Ref. [30] in our analyses. Clearly, the strong $\pi \eta$ final-state interaction plays the most important role in the $\gamma \gamma \rightarrow \pi \eta$ reaction around the $a_{0}(980)$ resonance region. Based on this argument, we use a similar expression to Eq. (12) to fit the $\pi \eta$ cross section, with different parameters $c_{1}^{\prime}$ and $c_{2}^{\prime}$, which mimic the $\pi \eta$ production mechanism in diphoton annihilation. The explicit formula to fit the $\gamma \gamma \rightarrow \pi \eta$ cross section reads

$$
\sigma(s)=\frac{\alpha^{2} q_{\pi \eta}}{2 s^{3 / 2}}\left|c_{1}^{\prime} D^{-1}(s)_{\pi \eta \rightarrow \pi \eta}+c_{2}^{\prime} D^{-1}(s)_{\pi \eta \rightarrow K \bar{K}}\right|^{2},
$$

with $\alpha$ the fine-structure constant. Analogously to Eq. (12), just one of the parameters $c_{1}^{\prime}$ and $c_{2}^{\prime}$ in Eq. (14) needs to be complex. We fix $c_{2}^{\prime}$ as a real parameter and treat $c_{1}^{\prime}$ as complex if required to improve the fit quality.

The phase shift and inelasticity can be easily read off from the $S$ matrix, which in our convention is related to the unitarized scattering amplitude $\mathcal{T}$ of Eq. (6) through

$$
S=1+2 i \sqrt{\rho(s)} \cdot \mathcal{T}(s) \cdot \sqrt{\rho(s)},
$$

with $\rho(s)=\sigma(s) /(16 \pi s)$. The phase shifts $\delta_{k k}$ and $\delta_{k l}$ and inelasticities $\varepsilon_{k k}$ and $\varepsilon_{k l}$, with $k \neq l$, are then given by

$$
S_{k k}=\varepsilon_{k k} \mathrm{e}^{2 i \delta_{k k}}, \quad S_{k l}=i \varepsilon_{k l} \mathrm{e}^{i \delta_{k l}} .
$$

\section{$2.2 U(3) \chi \mathrm{PT}$ in a finite volume}

Although the experimental $\pi \eta$ event distribution [29] and the $\gamma \gamma \rightarrow \pi \eta$ cross section [30] can provide some hints on the strong $\pi \eta$ interactions, both of them are complicated by the complex production mechanisms and cannot provide direct $\pi \eta$ scattering information. In fact, direct experimental measurements on the $\pi \eta$ scattering, such as the phase shifts and inelasticities, are still absent. This is one of the key obstacles that prevents a precise determination of the $a_{0}(980)$ properties.

Recently, the first calculation of $\pi \eta$ scattering, including the $K \bar{K}$ and $\pi \eta^{\prime}$ coupled channels, has been carried out in lattice QCD [17]. The simulations are done with three different lattice volumes, but only one large pion mass $\left(m_{\pi} \sim 391 \mathrm{MeV}\right)$ is used. By performing the analysis in many moving frames, a large number of discrete energy levels in three volumes are obtained. The rich spectra in a finite box contain direct information on the $\pi \eta$ scattering. In Ref. [17], a large number of different $K$-matrix parametrizations are used to extract the phase shifts and inelasticities from the various finite-volume energy levels. In this work, we propose to use another framework, the unitarized $U(3) \chi \mathrm{PT}$, to reanalyze the discrete spectra.

In order to use this approach to describe the lattice energy levels, we first need to include the finite-volume effects in unitarized $U(3) \chi \mathrm{PT}$. Generally speaking, there are two different kinds of volume dependence of the scattering amplitudes. First, there are the contributions which are

\footnotetext{
${ }^{4}$ Comparing with the formula in Refs. [16, 45 , we use a slightly different parameterization to fit the $\pi \eta$ event distribution in this work.
} 
exponentially suppressed $\propto \exp \left(-m_{P} L\right)$, where $m_{P}$ is the mass of the lightest particle in the problem at hand and $L$ denotes the size of the box. Second, if the energy is above threshold, there are contributions that are only power suppressed $\propto 1 / L^{3}$ and behave irregularly. It can be demonstrated (see, e.g., Refs. 46, 47]) that only the $s$-channel contributions can lead to the power-law corrections, while the crossed channels give rise only to the exponentially suppressed terms (there are exponentially suppressed $s$-channel contributions as well). This indicates that the power-suppressed contributions in the unitarized chiral amplitude in Eq. (6) are generated solely by the modification of the function $G(s)$, which incorporates the $s$-channel unitarity cut. On the contrary, the function $N(s)$, which contains the crossed-channel contributions by construction, contributes to the exponentially suppressed volume dependences only. In the present work, we include the important finite-volume effects through the function $G(s)$ in Eq. (6) and neglect the exponentially suppressed volume dependence of the $N(s)$ function. The same prescription has been used in the previous studies within the same framework [25[27, 48, 55].

Furthermore, we would like to comment on the relation of unitarized $\chi \mathrm{PT}$ in a finite volume with the Lüscher approach [18]. In fact, these two approaches are quite similar since, as can be easily shown, the finite-volume modification of the function $G(s)$ can be expressed through the Lüscher zeta function up to the exponentially suppressed contributions [25]. Thus, the only difference with the Lüscher approach amounts to the use of the different $K$-matrix parametrizations in the infinite volume: In the unitarized $\chi \mathrm{PT}$ case one effectively parameterizes the $K$ matrix through the solution of the coupled-channel equations, whereas simple algebraic parametrizations were used in Refs. [17,20,21]. If $L$ becomes smaller, the exponentially suppressed terms become important and these two approaches are no longer equivalent. However, we do not consider this case here.

Following the prescription in Ref. [25], the finite-volume effects can be implemented in the two-point loop function $G(s)$ in Eq. (7) by replacing the continuous three-momentum integral with the sum of allowed discrete momenta in the finite box with periodic boundary conditions. In order to perform the sum, it is convenient to integrate out the zeroth component of the four-momentum integral in Eq. (7). This gives

$$
G(s)^{\text {cutoff }}=\int^{|\vec{q}|<q_{\max }} \frac{\mathrm{d}^{3} \vec{q}}{(2 \pi)^{3}} I(|\vec{q}|),
$$

where

$$
\begin{aligned}
I(|\vec{q}|) & =\frac{w_{1}+w_{2}}{2 w_{1} w_{2}\left[E^{2}-\left(w_{1}+w_{2}\right)^{2}\right]}, \\
w_{i} & =\sqrt{|\vec{q}|^{2}+m_{i}^{2}}, \quad s=E^{2},
\end{aligned}
$$

and the ultraviolet three-momentum cutoff $q_{\max }$ is introduced to regularize the divergent integral. One could also use other regularization methods to obtain finite results, such as the dimensional regularization, cf. Eq. (8) , or include different types of form factors [25]. We use sharp cutoffs below. When calculating the function $G(s)$ in a finite box of length $L$ with periodic boundary conditions, one obtains

$$
\widetilde{G}=\frac{1}{L^{3}} \sum_{\vec{n}}^{|\vec{q}|<q_{\max }} I(|\vec{q}|)
$$


where a tilde on top of a symbol is introduced to denote the quantities in the finite volume and

$$
\vec{q}=\frac{2 \pi}{L} \vec{n}, \quad \vec{n} \in \mathbb{Z}^{3}
$$

The difference between the infinite- and finite-volume functions can then be calculated through

$$
\begin{aligned}
\Delta G & =\widetilde{G}-G^{\text {cutoff }} \\
& =\frac{1}{L^{3}} \sum_{\vec{n}}^{|\vec{q}|<q_{\max }} I(|\vec{q}|)-\int^{|\vec{q}|<q_{\max }} \frac{\mathrm{d}^{3} \vec{q}}{(2 \pi)^{3}} I(|\vec{q}|) .
\end{aligned}
$$

Note that the finite-volume correction $\Delta G$ is independent of the cutoff $q_{\max }$ in the limit $L \rightarrow \infty$ due to the cancellation of the cutoff dependences in the two terms in this equation, as explicitly demonstrated in Ref. [25]. In the practical calculation, we have explicitly verified that the cutoff dependence of $\Delta G$ is indeed quite small. In general, taking $q_{\max }=\frac{2 \pi}{L} n_{\max }$ and $L$ around $2 \mathrm{fm}$ $\left(m_{\pi} L \sim 4\right)$, the change of $\Delta G$ for the $\pi \eta$ channel is typically smaller than one percent when increasing $n_{\max }$ from 20 to 30 .

One can then add the finite-volume correction $\Delta G$ to the infinite-volume result $G^{\mathrm{DR}}$ in Eq. (8) to get the final expression of the $G$ function used in our study

$$
\widetilde{G}^{\mathrm{DR}}=G^{\mathrm{DR}}+\Delta G .
$$

This is the prescription followed in Ref. [55]. The expression $\widetilde{G}^{\mathrm{DR}}$ evaluated in the finite box should always be real in the whole energy region, which is guaranteed in Eq. (23) due to the cancellation of the imaginary parts in $G^{\mathrm{DR}}$ and the cutoff integral in $\Delta G$ above threshold.

The two-point loop function $G(s)$ in Eq. (77) is manifestly Lorentz invariant in the infinite volume. However, this is not the case for the finite-volume situation, where the Lorentz invariance is lost. One then needs to work out the explicit form of the loop function, when boosting from one frame to another. This issue has been addressed in Refs. [26, 56, 57] and we briefly recapitulate the main results in order to introduce the necessary notations.

In the CM frame of the two particles, one has $\vec{q}_{1}^{*}=-\vec{q}_{2}^{*}$, where we follow the convention that any quantity defined in the CM frame is marked with an asterisk. Now let us consider the two-particle system in a moving frame with total four-momentum $P^{\mu}=\left(P^{0}, \vec{P}\right)$. The square of the CM energy of the two-particle system is then given by $s=E^{2}=\left(P^{0}\right)^{2}-|\vec{P}|^{2}$. The three-momenta of the two particles in the moving frame are $\vec{q}_{1}$ and $\vec{q}_{2}=\vec{P}-\vec{q}_{1}$. Boosting to the CM frame, i.e., transforming $\vec{q}_{i=1,2}$ to $\vec{q}_{i=1,2}^{*}$, one straightforwardly obtains

$$
\vec{q}_{i}^{*}=\vec{q}_{i}+\left[\left(\frac{P^{0}}{E}-1\right) \frac{\vec{q}_{i} \cdot \vec{P}}{|\vec{P}|^{2}}-\frac{q_{i}^{0}}{E}\right] \vec{P} .
$$

Furthermore, following Ref. [26], we notice that one is free to impose the on-shell relation between energy and three-momentum: $q_{i}^{* 0}=\sqrt{\left|\vec{q}_{i}^{*}\right|^{2}+m_{i}^{2}}$. We also mention that this is equivalent to enforcing the on-shell condition for $q_{i}^{0}=\sqrt{\left|\vec{q}_{i}\right|^{2}+m_{i}^{2}}$, which automatically leads to the on-shell condition for $q_{i}^{* 0}$ through the following Lorentz transformation:

$$
q_{i}^{0}=\frac{q_{i}^{* 0} E+\vec{q}_{i} \cdot \vec{P}}{P^{0}} .
$$


In order to establish the relation of the functions $G(s)$ in the moving and CM frames, one needs to calculate the Jacobian of the transformation from $\mathrm{d}^{3} \vec{q}_{i}^{*}$ to $\mathrm{d}^{3} \vec{q}_{i}$. In this respect, it is convenient to rewrite Eq. (24), substituting Eq. (25). This gives

$$
\vec{q}_{i}^{*}=\vec{q}_{i}+\left[\left(\frac{E}{P^{0}}-1\right) \frac{\vec{q}_{i} \cdot \vec{P}}{|\vec{P}|^{2}}-\frac{q_{i}^{* 0}}{P^{0}}\right] \vec{P},
$$

where according to the on-shell condition for $q_{i=1,2}^{* 0}$ one has

$$
q_{1}^{* 0}=\frac{E^{2}+m_{1}^{2}-m_{2}^{2}}{2 E}, \quad q_{2}^{* 0}=\frac{E^{2}+m_{2}^{2}-m_{1}^{2}}{2 E} .
$$

Using Eq. (26) it is straightforward to obtain the Jacobian

$$
\int \mathrm{d}^{3} \vec{q}_{1}^{*}=\frac{E}{P^{0}} \int \mathrm{d}^{3} \vec{q}_{1}
$$

Then, the integral can be discretized through the following substitution

$$
\begin{array}{r}
\int^{\left|\vec{q}_{1}\right|^{*}<q_{\max }} \frac{\mathrm{d}^{3} \vec{q}_{1}^{*}}{(2 \pi)^{3}} I\left(\left|\vec{q}_{1}^{*}\right|\right) \Longrightarrow \\
\widetilde{G}^{\mathrm{MV}}=\frac{E}{P^{0} L^{3}} \sum_{\vec{q}_{1}}^{\left|\vec{q}_{1}^{*}\right|<q_{\max }} I\left(\left|\vec{q}_{1}^{*}\left(\vec{q}_{1}\right)\right|\right),
\end{array}
$$

with

$$
\begin{array}{ll}
\vec{q}_{1}=\frac{2 \pi}{L} \vec{n}, & \vec{n} \in \mathbb{Z}^{3}, \\
\vec{P}=\frac{2 \pi}{L} \vec{N}, & \vec{N} \in \mathbb{Z}^{3} .
\end{array}
$$

Note that the CM three-momentum of the two-particle system $\vec{P}$ in the finite box should only take the discrete values shown in Eq. (31) in order to impose the condition $\vec{q}_{1}+\vec{q}_{2}=\vec{P}$. The final $G$ function after taking into account the finite-volume corrections in the moving frame takes the form

$$
\widetilde{G}^{\mathrm{DR}, \mathrm{MV}}=G^{\mathrm{DR}}+\Delta G^{\mathrm{MV}}
$$

where

$$
\Delta G^{\mathrm{MV}}=\widetilde{G}^{\mathrm{MV}}-G^{\mathrm{cutoff}},
$$

with $G^{\mathrm{DR}}, G^{\text {cutoff }}$ and $\widetilde{G}^{\mathrm{MV}}$ given in Eqs. (즈), (17) and (29), respectively.

Before ending this section, we briefly comment on the partial wave mixing effects for a nonvanishing total momentum $\vec{P}$ in the finite box. A noticeable difference between $\vec{P}=0$ (CM frame) and $\vec{P} \neq 0$ (moving frame) is that, in the former case, the $S$ wave can mix with the $G$ wave only (the effect of such a mixing is presumed to be tiny), whereas in the latter case, there are more mixing patterns: Even the mixing of the $S$ and $P$ waves can not be excluded in general. The mixing terms between different partial waves could give some visible effects for some specific channels, such as the $\pi K S$ - and $P$-wave scattering, while in some other cases the mixing effects are tiny, such as the $\pi \pi S$ - and $D$-wave scattering [26]. Due to the fact that 
the isospin for the $P$-wave $\pi \pi$ is 1 and the isospin for $S$-wave $\pi \pi$ is 0 or 2 , there is no mixing between $\pi \pi S$ - and $P$-wave amplitudes.

The situation in $\pi \eta^{\left({ }^{\prime}\right)}$ and $K \bar{K}$ scattering is more subtle. The $G$ parity of $\pi \eta^{\left({ }^{\prime}\right)}$ scattering is definite and negative. There is no $P$-wave or higher odd waves in $K \bar{K}$ scattering with negative $G$ parity. Only even-wave $K \bar{K}$ scattering, such as $S$ and $D$ waves, can have negative $G$ parity. For the $P$-wave $\pi \eta^{\left({ }^{\prime}\right)}$ scattering, one has the $J^{P C}=1^{-+}$exotic quantum numbers and therefore one does not expect any strong interactions in the low energy region 5 . As for the $D$-wave $\pi \eta^{\left({ }^{\prime}\right)}$ scattering, it only starts to become important around the $a_{2}(1320)$ region, and it shows very little impact near the $K \bar{K}$ threshold, which is explicitly verified in the lattice simulations in Ref. [17]. Based on these arguments, it seems quite plausible that the mixing effects between the higher partial waves and the $S$ wave in $\pi \eta, K \bar{K}$ and $\pi \eta^{\prime}$ scattering are small. Therefore, in the present study we neglect the higher partial wave effects, which is explicitly verified to be a good assumption in Ref. [17].

In summary the formulas that we use to determine the lattice finite-volume energy levels are

$$
\operatorname{det}\left[I+N_{0}^{1}(s) \cdot \widetilde{G}^{\mathrm{DR}, \mathrm{MV}}\right]=0,
$$

for the moving frames, and

$$
\operatorname{det}\left[I+N_{0}^{1}(s) \cdot \widetilde{G}^{\mathrm{DR}}\right]=0,
$$

for the CM frame. The matrix $I$ in the previous two equations denotes the $3 \times 3$ unit matrix, and $\widetilde{G}^{\mathrm{DR}, \mathrm{MV}}$ and $\widetilde{G}^{\mathrm{DR}}$ should be understood as $3 \times 3$ diagonal matrices, with their matrix elements calculated for the $\pi \eta, K \bar{K}$ and $\pi \eta^{\prime}$ channels.

\section{Global fits to the lattice energy levels and experimental data}

In this section, we discuss the global fits to the lattice finite-volume energy levels and the experimental data, including a $\pi \eta$ event distribution [29] and the $\gamma \gamma \rightarrow \pi \eta$ cross section [30]. On the one hand, the lattice energy levels contain the direct $\pi \eta$ scattering information, but the numerical simulations are done with a relatively heavy pion mass around $391 \mathrm{MeV}$. On the other hand, the experimental data encode the $\pi \eta$ dynamics at physical masses, but both the event distribution and cross section of the diphoton fusion are affected by the complex production mechanisms, which usually bring additional uncertainties when extracting the direct $\pi \eta$ scattering information. Nonetheless, it is clear that the global fits to both kinds of data from lattice and experiment impose stronger constraints on the $\pi \eta$ scattering amplitudes than the fit to only one set of these data.

Concerning the lattice simulations, we focus on the energy levels below the $\pi \eta^{\prime}$ threshold and the data points considered in our fits are explicitly shown in Figs. 1 and 2. This amounts to 47 data points which are provided by the authors of Ref. [17 with the correlation information for those obtained within the same lattice volume. The data points considered in our work are exactly the same as those fitted in the two-channel formalism by using the various twochannel $K$-matrix parametrizations in Ref. [17. For the $\pi \eta$ event distribution, there are 11 data points [29], which are shown in Fig. 3. Note that the background parts given in Ref. [29] are explicitly extracted when we fit the event distribution. The $\gamma \gamma \rightarrow \pi \eta$ cross section points [30] are shown in Fig. 4 , which amounts to 10 more data points. The systematic error bands given in Ref. [30] are taken into account in the fits.

\footnotetext{
${ }^{5}$ It is also very unlikely that the possible exotic states $\pi_{1}(1400)$ and $\pi_{1}(1600)$ [1] will have important impact around the $K \bar{K}$ threshold region.
} 
For the fits to lattice energy levels, we take the masses for $\pi, K, \eta$ and $\eta^{\prime}$ from Ref. [17]

$m_{\pi}=391.3 \pm 0.7 \mathrm{MeV}, m_{K}=549.5 \pm 0.5 \mathrm{MeV}, m_{\eta}=587.2 \pm 1.1 \mathrm{MeV}, m_{\eta^{\prime}}=929.8 \pm 5.7 \mathrm{MeV}$.

For the fits to experimental data the values of the masses are the same as in Ref. [16]

$$
m_{\pi}=137.3 \mathrm{MeV}, \quad m_{K}=495.6 \mathrm{MeV}, \quad m_{\eta}=547.9 \mathrm{MeV}, \quad m_{\eta^{\prime}}=957.7 \mathrm{MeV} .
$$

The $\eta-\eta^{\prime}$ mixing angle in the flavor octet-singlet basis is needed in our theoretical model, as can be seen from Eq. (11). In order to calculate the LO $\eta-\eta^{\prime}$ mixing angle in Eq. (5), we first need to know the LO masses for the pion and kaon, i.e., $\bar{m}_{\pi}$ and $\bar{m}_{K}$. In the Appendix of Ref. [16], the explicit formulas are provided to calculate these two quantities and we do not quote the expressions here. Using the masses from the lattice simulations in Eq. (36), the LO $\eta-\eta^{\prime}$ mixing angle turns out to be

$$
\theta=(-10.0 \pm 0.1)^{\circ}
$$

which is in good agreement with the values given in Refs. 58, 59 and can be compared with the value $\theta^{\text {phys }}=-16.2^{\circ}$ at the physical masses [16]. Note that this mixing angle has also recently been calculated by using different lattice actions: see, e.g., Ref. [60].

Another important quantity that is needed in our calculation, as can be seen in Eq. (11), is the pion decay constant $F_{\pi}$. Its value at the specific masses of Eq. (36) is not reported in Ref. [17]. Therefore, we need to estimate $F_{\pi}$ at the unphysical masses within our approach. The one-loop $U(3) \chi \mathrm{PT}$ result is already given in Ref. [16], which reads

$$
\begin{aligned}
F_{\pi}=F\{1- & \frac{1}{16 \pi^{2} F^{2}}\left[m_{\pi}^{2} \ln \frac{m_{\pi}^{2}}{\mu^{2}}+\frac{m_{K}^{2}}{2} \ln \frac{m_{K}^{2}}{\mu^{2}}\right] \\
& \left.+\left[\frac{4 \widetilde{c}_{d} \widetilde{c}_{m}\left(m_{\pi}^{2}+2 m_{K}^{2}\right)}{F^{2} M_{S_{1}}^{2}}-\frac{8 c_{d} c_{m}\left(m_{K}^{2}-m_{\pi}^{2}\right)}{3 F^{2} M_{S_{8}}^{2}}\right]\right\} .
\end{aligned}
$$

In this equation, $\widetilde{c}_{m, d}$ and $c_{m, d}$ are the couplings of the $S U(3)$ singlet and octet bare scalar resonances with masses $M_{S_{1}}$ and $M_{S_{8}}$, which were introduced at the Lagrangian level. We take their values as determined in Ref. [16].

We point out that up to one-loop level precision there is an ambiguity in choosing the pion decay constant appearing inside the curly brackets on the right hand side (rhs) of Eq. (39). For example, one can also use the renormalized $F_{\pi}$ inside the curly brackets in this equation, since for a one-loop calculation the difference is of higher order. In order to conveniently deal with this ambiguity, we impose two extra conditions to determine the expression for $F_{\pi}$. The first condition is that one should recover the physical value of $F_{\pi}=92.4 \mathrm{MeV}$ when using the physical pion and kaon masses with a proper value of $F$. The other condition is that in the meantime we require that our extrapolation formula for $F_{\pi}$ reproduces other existing lattice simulation results [61 64], which were analyzed in Ref. [65] in a chiral framework, when using the specific masses in Eq. (36). Guided by these requirements, we find that when substituting $F=77.0 \mathrm{MeV}$ in Eq. (39), we get the correct value for $F_{\pi}$ with physical pion and kaon masses, while taking the masses in Eq. (36) leads to $F_{\pi}=105.9 \mathrm{MeV}$, a value that is reasonably close to other lattice simulation results 6164. Therefore, we take $F_{\pi}=105.9 \mathrm{MeV}$ as the central value in our fits to the lattice energy levels of Ref. [17].

However, in order to make a further test about the influence of using different $F_{\pi}$ extrapolation forms on the extracted energy levels, we also replace $F$ inside the curly brackets in 
Eq. (39) with the physical value of $F_{\pi}$. In this case we find that with $F=81.1 \mathrm{MeV}$ the rhs of Eq. (39) leads to $F_{\pi}=92.4 \mathrm{MeV}$ at physical pion and kaon masses, and then it predicts $F_{\pi}=102.3 \mathrm{MeV}$ with the masses in Eq. (36). We consider the differences of $F_{\pi}$ obtained with the two different extrapolation forms as an additional source of uncertainty in our study, which can be treated as a systematic error. In summary at the masses given in Eq. (36) we use

$$
F_{\pi}=105.9 \pm 3.6 \mathrm{MeV}
$$

to extract the finite-volume energy levels. Comparing with the lattice results given in Refs. 6164, we may conclude that our estimate of the error in $F_{\pi}$, Eq.(40), is quite conservative. When fitting to experimental data, we always fix $F_{\pi}$ at its physical value.

\subsection{Leading-order fit}

In this part we present the LO fit results. The $N_{0}^{1}(s)$ matrix function in Eq. (10) is simply given by the LO $T_{0}^{1}(s)^{(2)}$ in Eq. (11). At this order the only unknown parameters in the unitarized chiral amplitudes are the three subtraction constants $a_{\pi \eta}, a_{K \bar{K}}$ and $a_{\pi \eta^{\prime}}$. The fits turn out to be rather insensitive to the value of $a_{\pi \eta^{\prime}}$ (a feature that is also seen in the NLO fits discussed in the next section). Therefore we will always fix its value to be equal to $a_{K \bar{K}}$, both in the LO and NLO fits. Furthermore in the LO fit we find that just one common subtraction constant for the three channels is already enough to obtain a good fit. Leaving the value for $a_{K \bar{K}}$ free barely improves the fit quality. Therefore we impose $a_{\pi \eta}=a_{K \bar{K}}=a_{\pi \eta^{\prime}}$ for this case. As discussed in Sec. 2.1 we need to include additional parameters in order to describe a $\pi \eta$ event distribution and the $\gamma \gamma \rightarrow \pi \eta$ cross section, cf. Eqs. (12) and (14). For the $\pi \eta$ event distribution, two real parameters $c_{1}$ and $c_{2}$ are found to be enough to reproduce the data well. For the $\gamma \gamma \rightarrow \pi \eta$ cross section, we find that just one real parameter $c_{2}^{\prime}$ alone is able to give reasonable description of the experimental data, and take $c_{1}^{\prime}=0$.

The LO fit gives a reasonable description of the overall data, with a $\chi^{2} /$ d.o.f $=104.5 /(68-$ $4) \simeq 1.69$. The chi square contributed by the 47 lattice energy levels is 90.2 , and the chi square from the 21 experimental data is 14.3. Note that the correlation information among the lattice energy levels within the same volume [17] is considered in our fit. The value of the subtraction constant from this fit is

$$
a_{\pi \eta}=-1.44 \pm 0.15 \pm 0.01 .
$$

The values for the phenomenologically motivated parameters $c_{1}, c_{2}$ and $c_{2}^{\prime}$ are $c_{1}=0.44 \pm$ $0.14 \pm 0.00 \mathrm{MeV}^{-1}, c_{2}=-0.27 \pm 0.11 \pm 0.00 \mathrm{MeV}^{-1}$ and $c_{2}^{\prime}=2.18 \pm 0.36 \pm 0.02$. The first error bar of each parameter corresponds to the statistical one and the second one is caused by the uncertainties of the unphysical masses in Eq. (36). Note that when using Eq. (14) to fit the $\gamma \gamma \rightarrow \pi \eta$ cross section, we have introduced the proper normalization factor to transform the unit $\mathrm{MeV}^{-2}$ to nanobarn. The statistical error bars of the parameters $a_{\pi \eta}, c_{1}, c_{2}$ and $c_{2}^{\prime}$ are calculated in the following way. We randomly vary the parameters around their central values from the best fit, recalculate the corresponding new chi square and then only keep the ones that give $\chi^{2} \leq \chi_{0}^{2}+\sqrt{2 \chi_{0}^{2}}$ (with $\chi_{0}^{2}$ the chi-square value from the best fit), i.e. those within the 1- $\sigma$ standard deviation. In order to estimate the influences on the parameters from the uncertainties of the unphysical masses in Eq. (36), we have performed a large number of fits by randomly varying the masses within uncertainties. It turns out that the variances of the central values of the fitted parameters are one order smaller than the statistical error bars and hence negligible. With those parameter configurations within $1-\sigma$ uncertainty, we also calculate the 

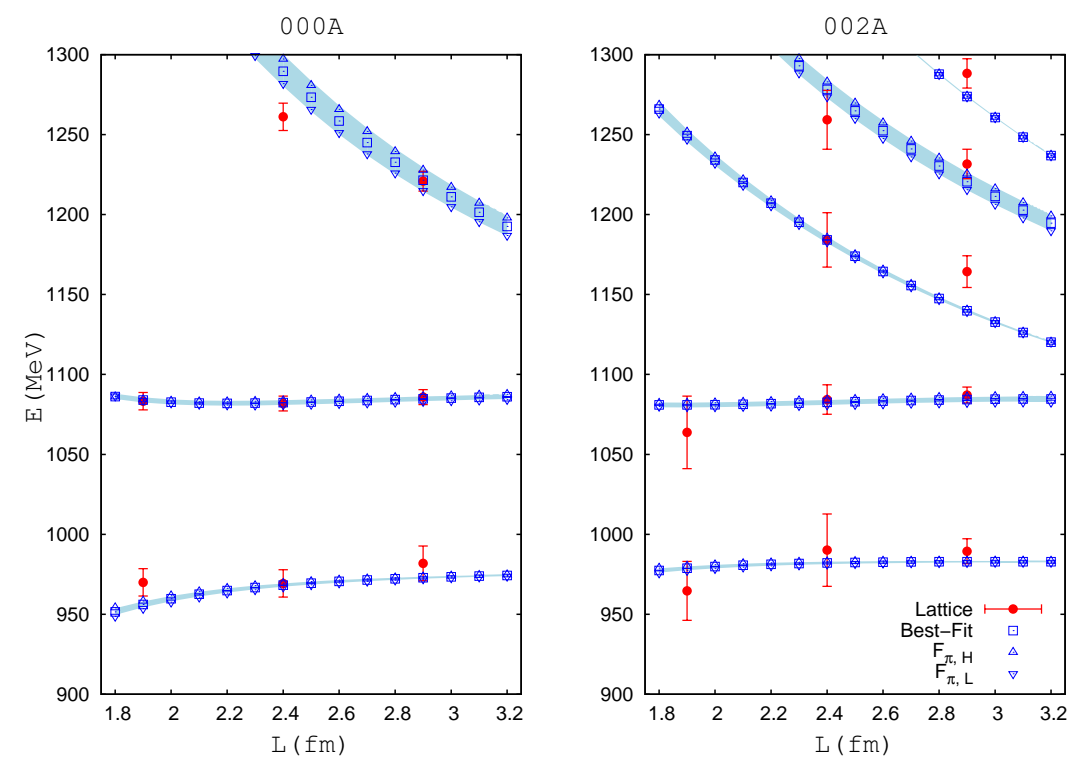

Figure 1: Fit results of the finite-volume energy levels for the ensembles $000 A$ and $002 A$ at leading order. The lattice data are taken from Ref. [17]. The square symbols represent the results from our best fit and the shaded areas correspond to the 1- $\sigma$ uncertainties. The upwards and downwards triangle symbols, labeled as $F_{\pi, \mathrm{H}}$ and $F_{\pi, \mathrm{L}}$, denote the results obtained by taking the upper and lower limits of $F_{\pi}$ in Eq. (40).

error bands of the other quantities, including the finite-volume energy levels, event distribution, cross section, phase shifts, inelasticities, the pole positions, and corresponding residues.

The reproduction of the lattice energy levels is shown in Figs. 1 and 2, where the square symbols stand for the results from our best fit and the shaded areas correspond to the $1 \sigma$ error bands. The upwards and downwards triangle symbols denote the results calculated for the upper and lower limits of $F_{\pi}$ in Eq. (40). The fit results for the $\pi \eta$ event distribution and $\gamma \gamma \rightarrow \pi \eta$ cross section are given in Figs. 3 and 4 , respectively. The LO best fits are plotted in blue by the dotted lines, and their hatched surrounding areas present the $1 \sigma$ uncertainties as explained before.

It is interesting to discuss two different variants of LO fits. In one case, we study the influences of using different pNGB decay constants in the scattering amplitudes in Eq. (11). Although distinguishing different pNGB decay constants is beyond the LO accuracy, it may cause a visible effect when performing the chiral extrapolation. Here we do a tentative study of this effect by only distinguishing the kaon decay constant $F_{K}$ from the others, due to the prominent $K \bar{K}$ threshold enhancement around the $a_{0}(980)$ energy region. To be more specific, we replace one $F_{\pi}$ in the $\pi \eta \rightarrow K \bar{K}, K \bar{K} \rightarrow \pi \eta^{\prime}$ amplitudes in Eq. (11) by $F_{K}$ and replace $F_{\pi}^{2}$ in $K \bar{K} \rightarrow K \bar{K}$ by $F_{K}^{2}$. At the physical mass, $F_{K}$ is set to $110.1 \mathrm{MeV}$ [1] and when taking the unphysical masses in Eq. (36) $F_{K}$ is fixed to $115.0 \mathrm{MeV}$, a value consistent with the previous lattice determinations 61 64. We mention that using a somewhat different value for $F_{K}$ at the unphysical meson masses only moderately changes our discussion below, since its effect can be compensated by slightly adjusting the subtraction constants. The resulting parameters from the fit are $a_{\pi \eta}=-1.73 \pm 0.16, c_{1}=0.31 \pm 0.14, c_{2}=-0.32 \pm 0.11$ and $c_{1}^{\prime}=2.13 \pm 0.36$, with $\chi^{2} /$ d.o.f $=107.1 /(68-4)$. Even though the subtraction constant is decreased by about $20 \%$ 

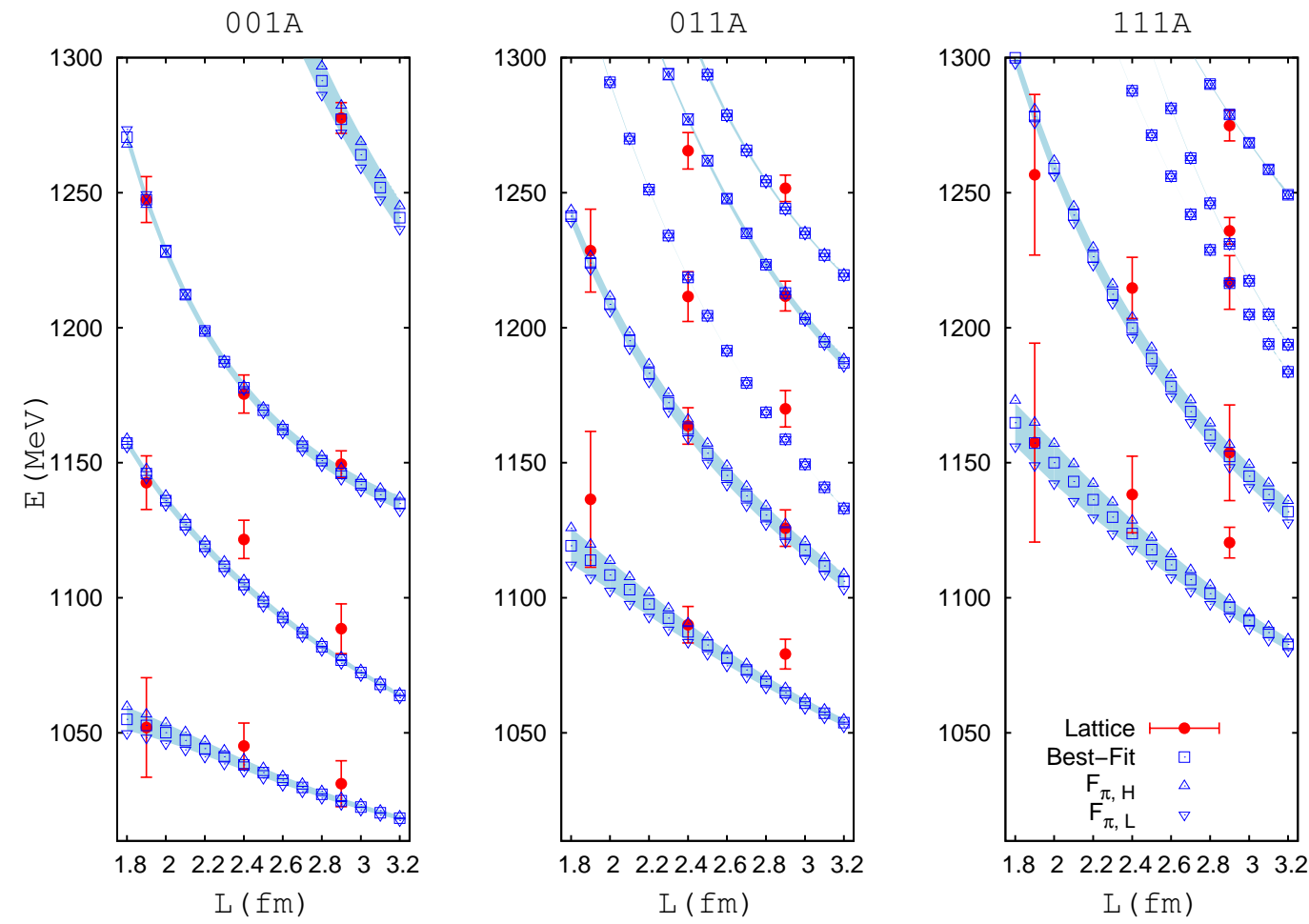

Figure 2: Fit results of the finite-volume energy levels for the ensembles $001 A, 011 A$ and $111 A$ at leading order. The lattice data are taken from Ref. [17. For notations, see Fig. 1.

due to the replacement of $F_{\pi}$ by $F_{K}$ in the amplitudes involving kaon, we will show in Secs. 4 and 5 that the fits using different meson decay constants lead to qualitatively similar phase shifts and inelasticities to the case with a common $F_{\pi}$ in all amplitudes. In the other case, we perform the fit by only including the lattice energy levels. The resulting parameter turns out to be $a_{\pi \eta}=-1.49 \pm 0.20$, with $\chi^{2} /$ d.o.f $=89.3 /(47-1)$. The value obtained here is perfectly compatible with the result in Eq. (41) from the global fit by simultaneously including both the lattice and experimental data. In other words, the LO expression can give a consistent descriptions of both the lattice data with large unphysical masses and the experimental data with physical masses.

\section{$3.2 \quad$ Next-to-leading-order fit}

As demonstrated in the previous section and also in many earlier papers [4, 6, 7, 66, 67, the LO unitarized chiral amplitudes can already reasonably describe the various $\pi \eta$ reactions around the $K \bar{K}$ threshold energy region. As a result, it is reasonable to require that including the higher order effects in the unitarized amplitudes should not spoil the LO results. Therefore, as a first step to perform the NLO fits, we impose the condition that the NLO unitarized chiral amplitudes stay close to the LO results within a $20 \%$ uncertainty around the $K \bar{K}$ threshold. This condition - in addition to fitting the lattice energy levels, $\pi \eta$ event distribution and the $\gamma \gamma \rightarrow \pi \eta$ cross section - stabilizes the fit, given the numerous free parameters. After obtaining good fits, we finally release the closeness condition of the LO and NLO amplitudes. We find that in this way the fit is stable, and the final NLO amplitudes still qualitatively resemble the 


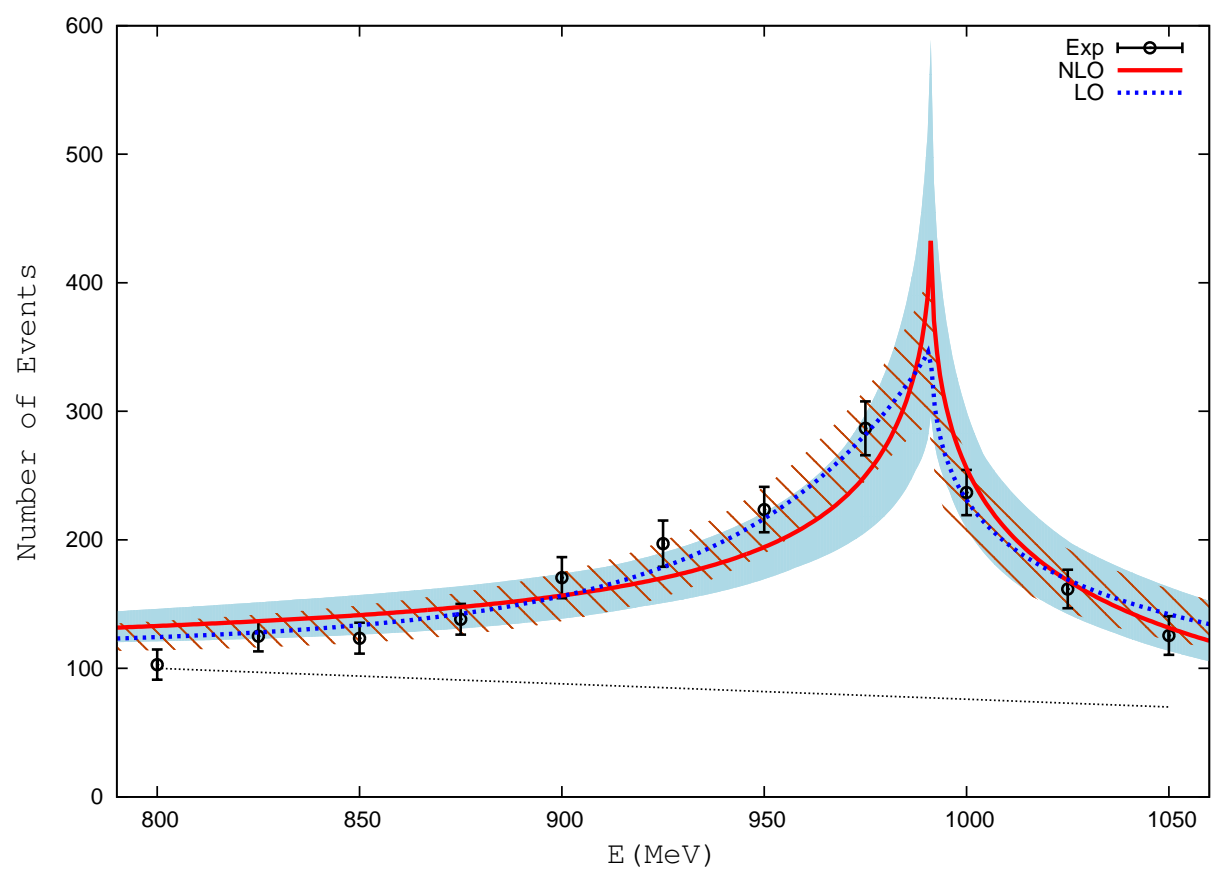

Figure 3: The $\pi \eta$ event distribution. The experimental data are taken from Ref. [29]. The black dotted line represents the background extracted from this reference. The blue dotted and solid lines denote the LO and NLO fit results, respectively. The shaded areas correspond to the $1 \sigma$ error bands.

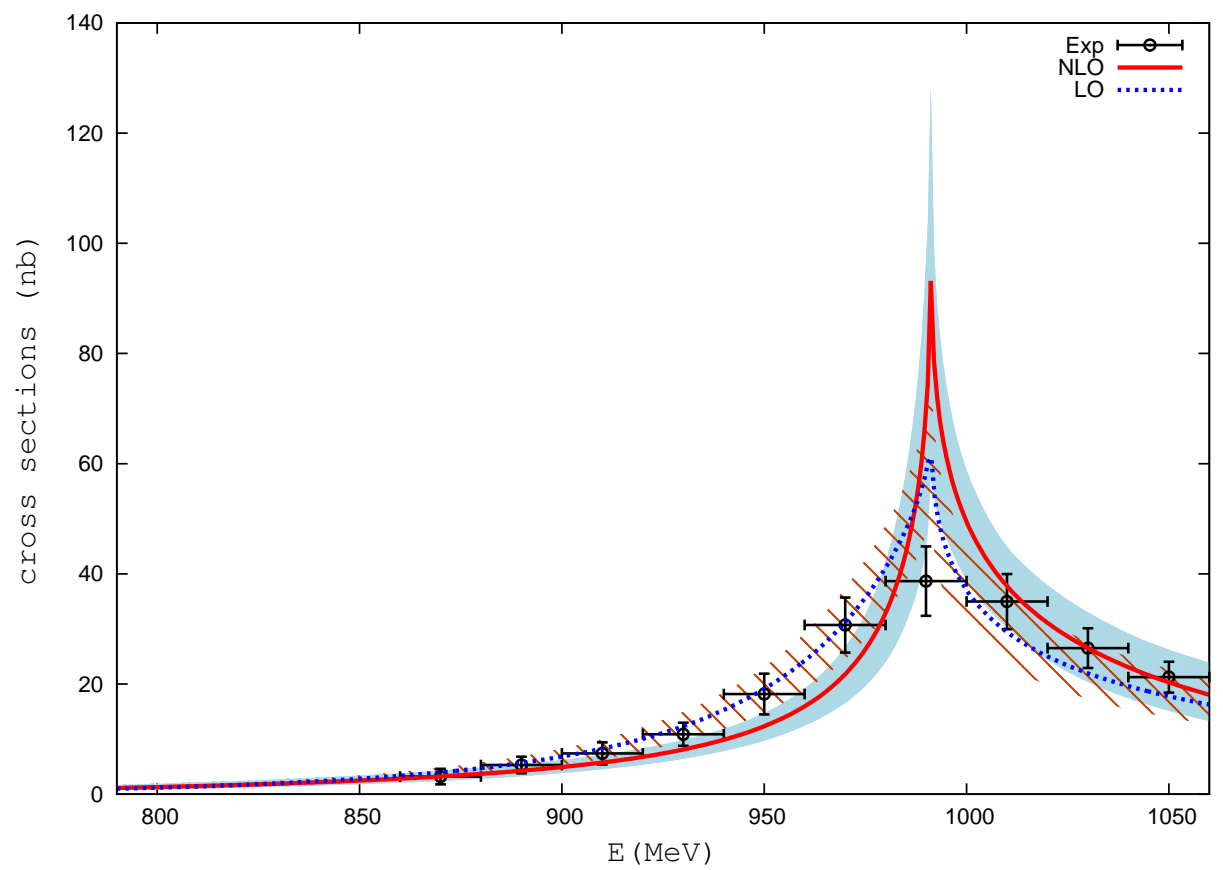

Figure 4: Cross section of $\gamma \gamma \rightarrow \pi \eta$. The experiment data are taken from Ref. [30] and the systematic error bands from this reference are included in our fits. For notations, see Fig. 3 . 


\section{LO ones.}

There are more parameters in the NLO unitarized chiral amplitudes than in the LO ones. We fit the three subtraction constants $a_{\pi \eta}, a_{K \bar{K}}$ and $a_{\pi \eta^{\prime}}$, which appear in the $\pi \eta, K \bar{K}$ and $\pi \eta^{\prime}$ channels. The other parameters are already well determined in Ref. [16] and we take the values therein. At NLO, we find that it is impossible to obtain a good fit with just one subtraction constant. Both $a_{\pi \eta}$ and $a_{K \bar{K}}$ are fitted in this case, while the fits are quite insensitive to $a_{\pi \eta^{\prime}}$; thus, we simply fix its value to the one of $a_{K \bar{K}}$. For the additional parameters mimicking the $\pi \eta$ production mechanisms in Eqs. (12) and (14), it turns out that with real $c_{1}$ and $c_{2}$ we are able to give a good description of the event distribution, and with $c_{2}^{\prime}$ alone one can reasonably reproduce the cross section. We verify that freeing the parameter $c_{1}^{\prime}$ barely changes the fit quality. Therefore we fix $c_{1}^{\prime}=0$ as in the LO case.
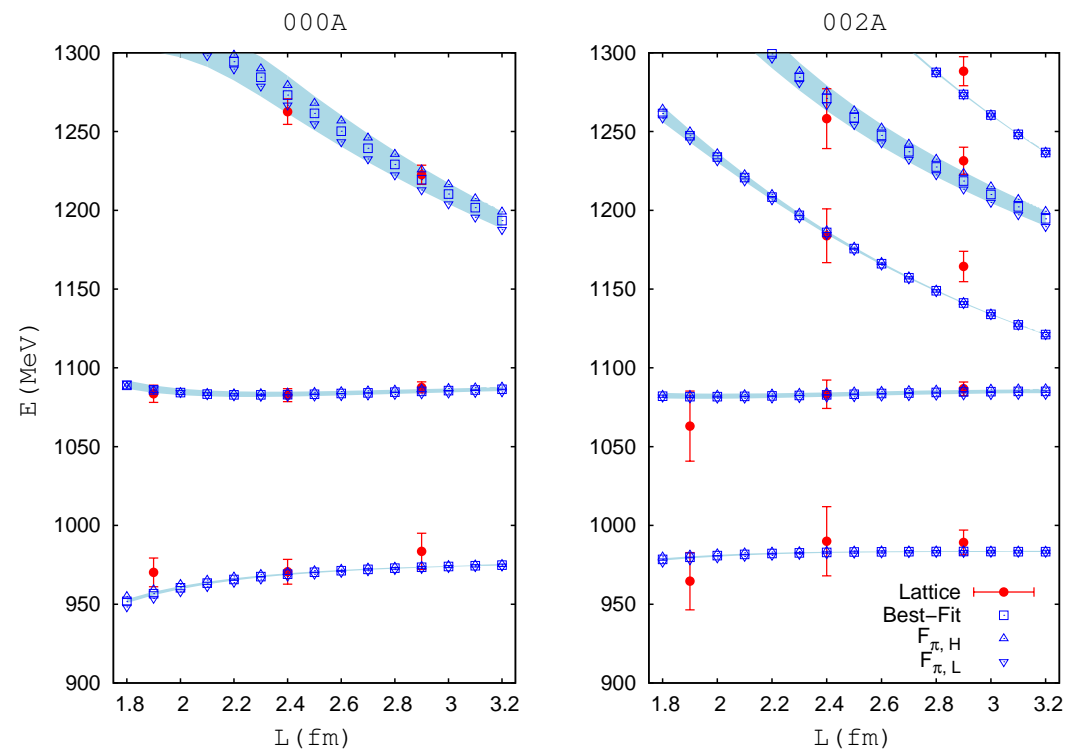

Figure 5: Fit results of the finite-volume energy levels for the ensembles $000 A$ and $002 A$ at next-to-leading order. For notations, see Fig. 1.

The best NLO fit gives $\chi^{2} /$ d.o.f $=105.4 /(68-5) \simeq 1.67$, among which 72.7 is contributed by the lattice energy levels. The values of the two subtraction constants are

$$
a_{\pi \eta}=0.56 \pm 0.90 \pm 0.05, \quad a_{K \bar{K}}=-1.62 \pm 0.33 \pm 0.02 .
$$

Note that within errors the present determination of the subtraction constant $a_{\pi \eta}$ agrees with the value in Ref. [16], which gives $a_{\pi \eta} \simeq 2 \pm 3$. The subtraction constant $a_{K \bar{K}}$ in $I J=10$ scattering in Ref. [16] was simply taken from the $I J=00 \pi \pi$ scattering: $a_{K \bar{K}} \simeq-1.15 \pm 0.1$, by invoking $S U(3)$ symmetry. The values for the parameters related to the $\pi \eta$ production are: $c_{1}=0.48 \pm 0.16 \pm 0.01 \mathrm{MeV}^{-1}, c_{2}=-0.34 \pm 0.10 \pm 0.00 \mathrm{MeV}^{-1}$ and $c_{2}^{\prime}=2.22 \pm 0.50 \pm 0.01$. The first error bar of each parameter is statistical and the second one is given by the uncertainties of the unphysical masses in Eq. (36). The error bars of the parameters are calculated in the same way as explained in the LO case. The values of the parameters involved in the $\pi \eta$ production reactions turn out to be rather similar to their LO values, indicating that the $D$ functions in Eq. (12) for both fits corresponding to the $a_{0}(980)$ are not very different. It is worth pointing out that we have tried to fit only the lattice energy levels with the NLO unitarized amplitudes. 

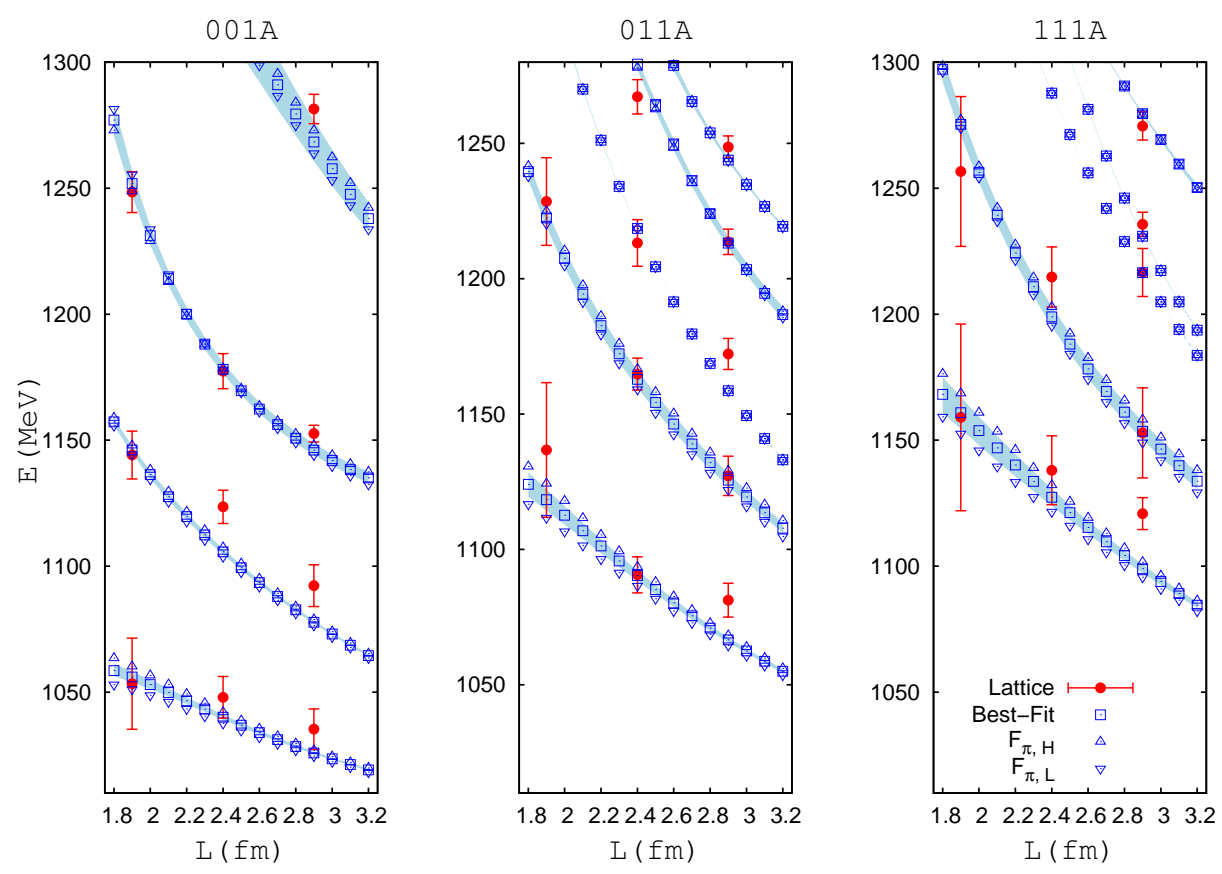

Figure 6: Fit results of the finite-volume energy levels for the ensembles $001 A, 011 A$ and $111 A$ at next-to-leading order. For notations, see Fig. 1 .

Though the $\chi^{2}$ from the lattice energy levels in this case decreases around 10 compared with the corresponding value from the global fit, the resulting NLO amplitudes turn out to be rather different from the LO ones and they give unsatisfactory descriptions of the experimental $\pi \eta$ event distribution data within the simple formalism in Eq. (12), even with the complex $c_{1}$ parameter. Moreover, the well-established $a_{0}(1450)$ resonance, which is explicitly introduced in the NLO amplitude, is also strongly distorted, i.e. far away from its PDG value [1], which hints that the NLO fit to only lattice energy levels does not seem to correspond to the real physical solution. Therefore we refrain from discussing this fit further and focus on the global fit by simultaneously including the lattice and the experimental data. In this respect, we argue that the lattice energy levels with smaller pion masses can be quite useful to further constrain the NLO amplitudes. This is because unlike the description of the complicated $\pi \eta$ production, no additional theoretical uncertainties will be introduced to study the lattice energy levels, as they are purely determined by the $\pi \eta$ scattering.

The NLO fit results for the lattice energy levels are given in Figs. 5]and 6. The reproduction of the $\pi \eta$ event distribution and $\gamma \gamma \rightarrow \pi \eta$ cross section is shown in Figs. 3 and 4 respectively, together with the LO results. The meaning of the symbols used in the LO figures is kept for the NLO ones. Though the overall reproduction of the lattice energy levels and experimental data is quite similar for the LO and NLO fits, the latter gives a slightly better description of the 47 lattice energy levels with $\chi^{2}=72.7$ than the former case with $\chi^{2}=90.2$. The LO fit yields better results for the $\pi \eta$ event distribution and the $\gamma \gamma \rightarrow \pi \eta$ cross section. In other words, the NLO unitarized amplitude seems to work better for the $\pi \eta$ dynamics at large unphysical masses, while the LO amplitude seems more efficient to reproduce the experimental peaks around the $a_{0}(980)$ region. Nevertheless, this statement should be taken with a grain of salt, because many parameters in the NLO amplitudes are determined from other processes 
in Ref. [16] and they can not be solely fixed by the current available data from $\pi \eta$ scattering. A more refined theoretical model to describe complicated $\pi \eta$ production mechanisms to fit experimental data might also be useful to further discern the two amplitudes.

\section{Phase shifts, inelasticities and poles at unphysical masses}
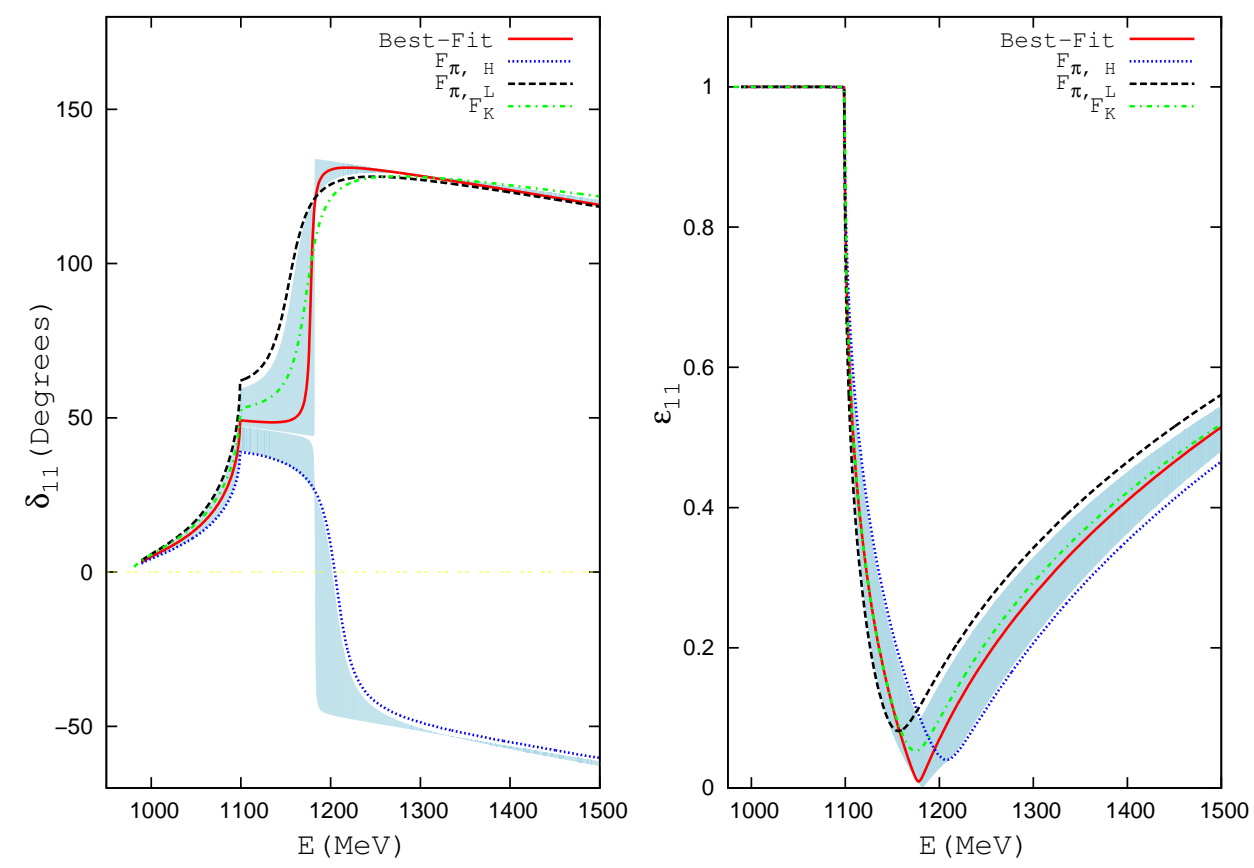

Figure 7: Leading-order results for phase shifts and inelasticities for $\pi \eta \rightarrow \pi \eta$ scattering with the unphysical masses for the $\pi, K, \eta$ and $\eta^{\prime}$ given in Eq. (36). The best-fit results are plotted as red solid lines and the shaded areas represent the $1 \sigma$ uncertainties. The blue dotted (denoted by $F_{\pi, \mathrm{H}}$ ) and the black dashed (denoted by $F_{\pi, \mathrm{L}}$ ) lines show the results by taking the upper and lower limits of $F_{\pi}$ in Eq. (40), respectively. The green dashed-dotted lines correspond to the results by distinguishing between $F_{\pi}$ and $F_{K}$ in the scattering amplitudes. See the text for more details.

After fixing the parameters in the unitarized chiral amplitudes, we calculate the phase shifts, inelasticities, resonance poles and their residues with the unphysical masses used in Ref. [17. In Fig. 7, we give our LO predictions for the $\pi \eta$ scattering phase shifts and inelasticities. We observe two different kinds of solutions for the phase shifts within $1 \sigma$ uncertainty. Both of them show a clear kink structure at the $K \bar{K}$ threshold. On the one hand, for the first set of solutions we see that the phase shifts show a steep increase around $1.2 \mathrm{GeV}$ and are always positive. We explicitly verify that this solution corresponds to the situation when $a_{\pi \eta}<-1.41$. Our best fit and the result with a lower limit of $F_{\pi}$ in Eq. (40), shown with red solid and black dashed lines in Fig. 7, respectively, belong to this kind of solution. On the other hand, in the second case when $a_{\pi \eta}>-1.41$ the phase shifts exhibit mild and continuous changes with increasing energies and become negative in the energy region above $1.2 \mathrm{GeV}$. The result obtained with the upper limit of $F_{\pi}$ in Eq. (40) is similar to the second case. It is also interesting to note that the phase shifts obtained in the lattice analyses in Ref. [17] are similar to our second type 

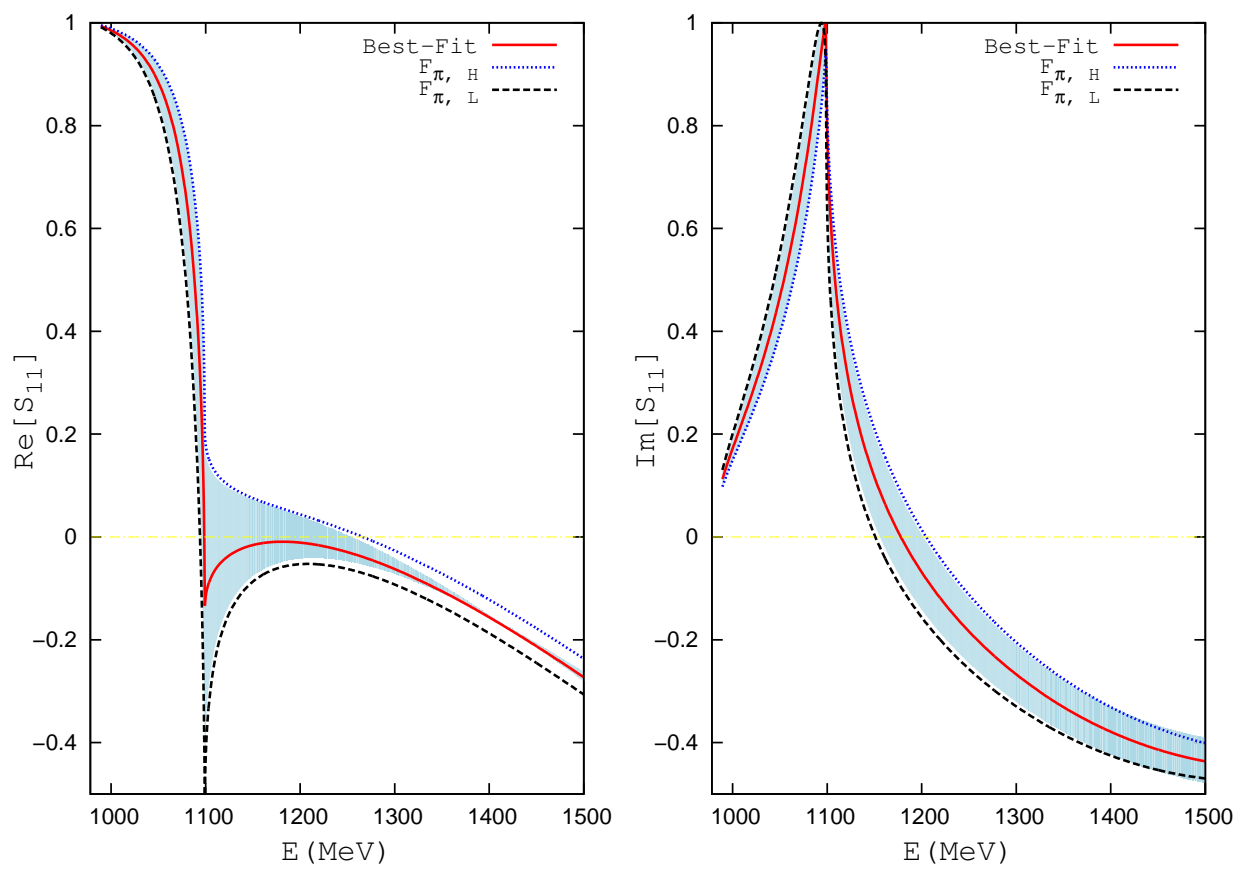

Figure 8: The $S$ matrix defined in Eq. (16) for $\pi \eta \rightarrow \pi \eta$ scattering at leading order with heavy unphysical meson masses in Eq. (36). The left panel is for the real part, and the right panel is for the imaginary part. For notations, see Fig. 7 .

of solution, i.e., to the lower branch shown in the left panel of Fig. 7 . In the right panel, we give the inelasticity of the $\pi \eta$ scattering. Below the $K \bar{K}$ threshold, the inelasticity is equal to 1 , as it should be. At the $K \bar{K}$ threshold the inelasticity suddenly decreases to almost zero and gradually increases when the energy becomes larger. As shown in Fig. 7, the inelasticities show a qualitatively similar behavior within 1- $\sigma$ uncertainty and with different extrapolation forms of $F_{\pi}$. On physical grounds, both types of solutions for the phase shifts are indeed very similar, since above $1.2 \mathrm{GeV}$ both results for the $\pi \eta$ phase shifts only differ by 180 degrees. Although in the energy region between the $K \bar{K}$ threshold and $1.2 \mathrm{GeV}$ the phase shifts show large uncertainties, the inelasticity in this region is almost zero. In order to clearly demonstrate the similarity of the underlying dynamics between these two different branches of phase shifts in Fig. 7, we give the $S$ matrix for the $\pi \eta \rightarrow \pi \eta$ scattering in Fig. 8 . This also indicates that the $\pi \eta \rightarrow K \bar{K}$ scattering plays a more important role in this specific energy range. In Fig. 9 , we show the LO phase shifts (left panel) and inelasticities (right panel) for the $\pi \eta \rightarrow K \bar{K}$ scattering with blue dashed lines. Note that, as expected, this transition amplitude just varies slightly within the $1 \sigma$ region. It is worth pointing out that around the $K \bar{K}$ threshold the $\pi \eta$ phase shifts are also found to be quite sensitive to the small variation of parameters in Ref. [17], but the scattering amplitudes are stable.

Our NLO predictions for the $\pi \eta$ scattering phase shifts and inelasticities calculated at the unphysical masses of Eq. (36) are shown in Fig. 10, Similarly to the LO case, two different kinds of solutions for the phase shifts within 1- $\sigma$ uncertainty are found. The first set of fits for the phase shifts shows a steep increase around $1170 \mathrm{MeV}$ and are always positive. The result with the lower limit of $F_{\pi}$ in Eq. (40), shown by the black dashed line in Fig. 10 belongs to this kind of solution. Most of the parameter configurations of the NLO fits lead to the second 

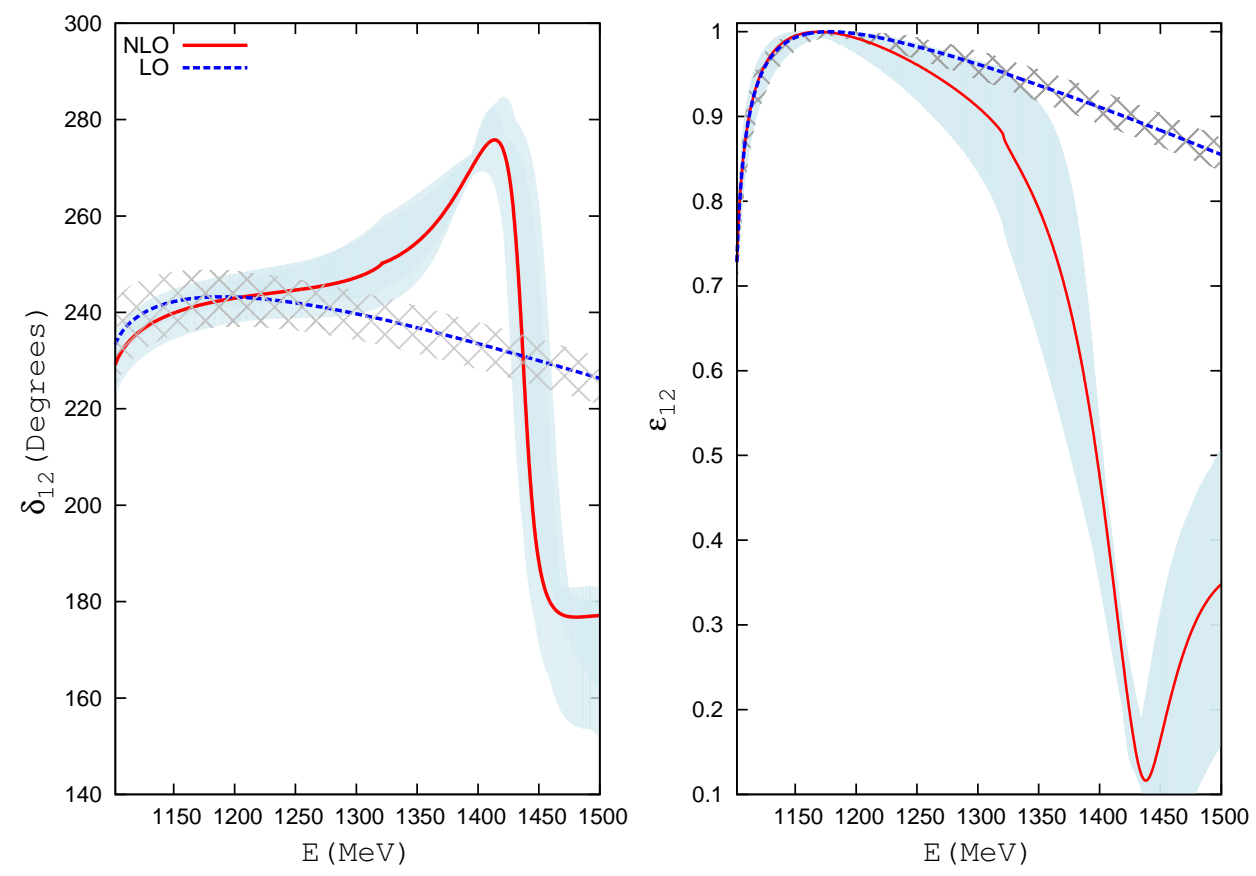

Figure 9: Phase shifts and inelasticities for $\pi \eta \rightarrow K \bar{K}$ scattering with the heavy unphysical masses for $\pi, K, \eta$ and $\eta^{\prime}$ in Eq. (36). The left panel is for the phase shifts and the right one is for inelasticities. Both the LO (blue dashed lines) and NLO (solid red lines) results are given. The shaded areas represent the 1- $\sigma$ uncertainties.
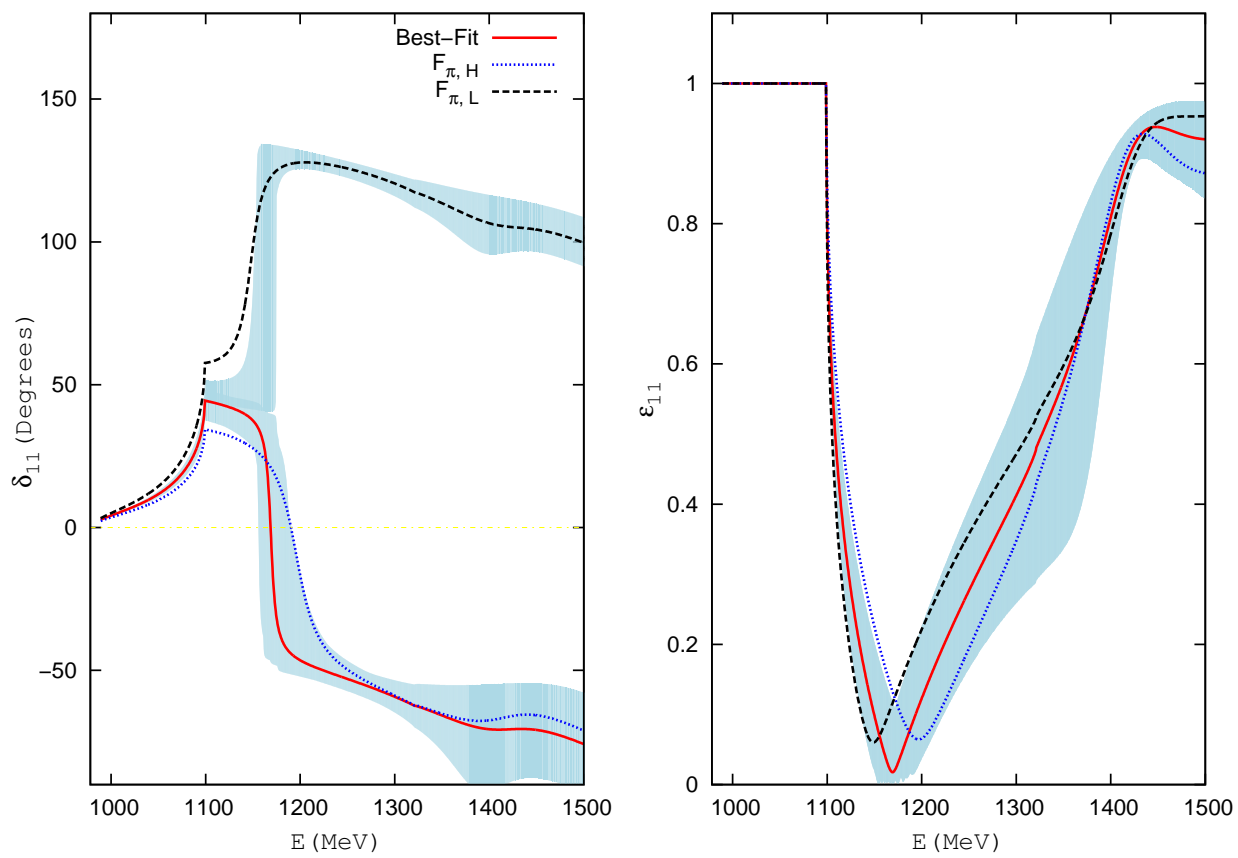

Figure 10: NLO results for phase shifts and inelasticities for $\pi \eta \rightarrow \pi \eta$ scattering with the unphysical masses for $\pi, K, \eta$ and $\eta^{\prime}$ in Eq. (36). For notations, see Fig. 7. 

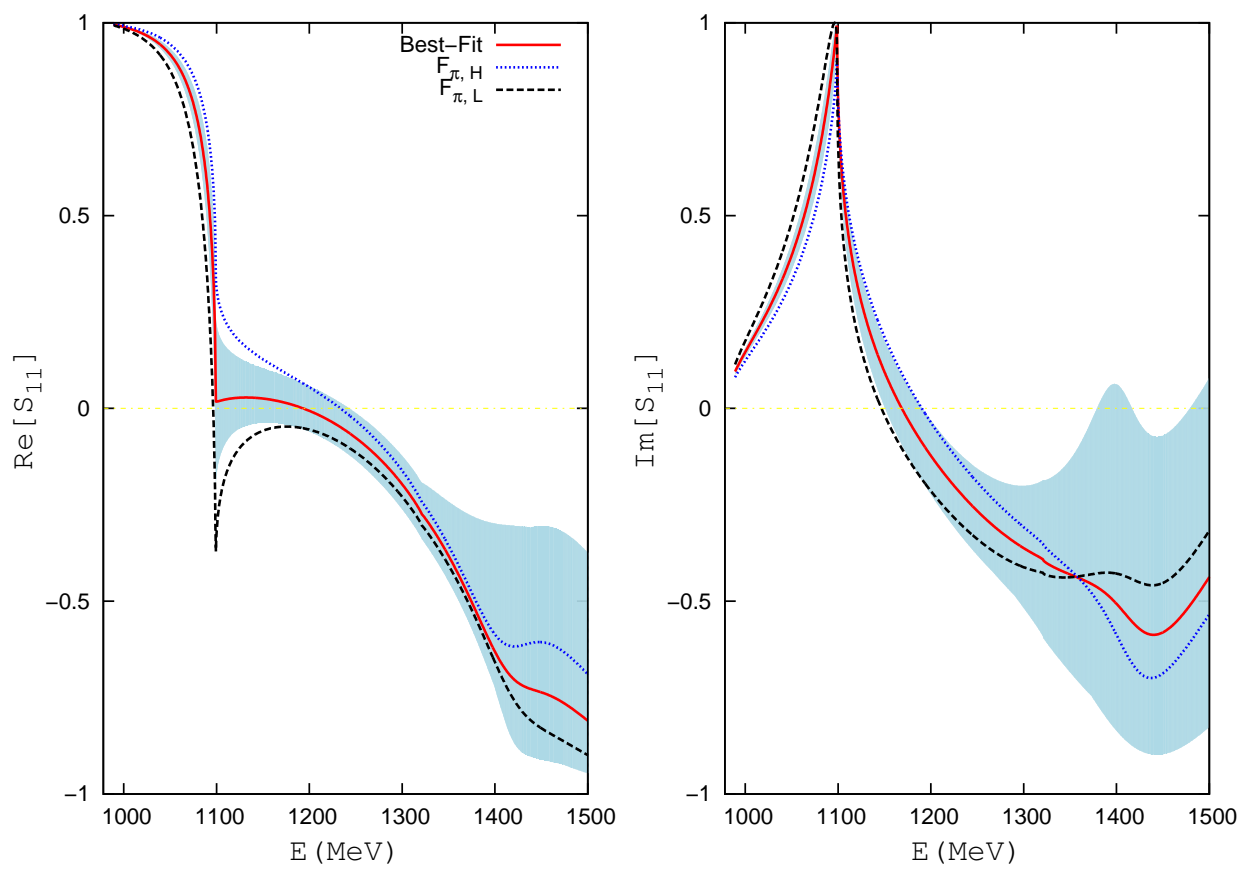

Figure 11: The $S$ matrix defined in Eq. (16) for $\pi \eta \rightarrow \pi \eta$ scattering at next-to-leading order with heavy unphysical meson masses in Eq. (36). The left (right) panel is for the real (imaginary) part. For notations, see Fig. 7.

type of solution: The phase shifts exhibit mild and continuous changes with increasing energies and become negative in the energy region above $1170 \mathrm{MeV}$ or so. The result obtained with the upper limit of $F_{\pi}$ in Eq. (40) belongs to the second type of solution. The inelasticities of the $\pi \eta$ scattering are given in the right panel of Fig. 10. The NLO inelasticities show a different behavior compared to the $\mathrm{LO}$ ones above the $1.3 \mathrm{GeV}$ region. There is a rapid increase in the NLO case around $1.4 \mathrm{GeV}$. The reason behind this behavior is that in the NLO amplitude the $a_{0}(1450)$ resonance is explicitly included, while only the lowest order contact meson-meson interactions are incorporated at LO and the $a_{0}(1450)$ can not be generated in this case. Similar to the LO situation, the phase shifts above $1.2 \mathrm{GeV}$ only differ by 180 degrees, but show large uncertainties in the energy range between the $K \bar{K}$ threshold and $1.2 \mathrm{GeV}$. However, these large uncertainties do not matter since the inelasticities are very small in the same region. This statement can be clearly seen in Fig. 11, where the real and imaginary parts of the $S$ matrix for $\pi \eta \rightarrow \pi \eta$ scattering at NLO are displayed. Then the physics is dominated by the $\pi \eta \rightarrow K \bar{K}$ scattering in this region. The NLO phase shifts and inelasticities for $\pi \eta \rightarrow K \bar{K}$ scattering, together with the LO results, are given in Fig. 9. One can clearly see that the LO and NLO phase shifts and inelasticities for $\pi \eta \rightarrow K \bar{K}$ scattering are quite similar in the range from the $K \bar{K}$ threshold up to around $1.3 \mathrm{GeV}$, somewhat before the effects of the $a_{0}(1450)$ resonance become dominant.

A unique way to characterize a resonance is to look for the corresponding poles in the complex energy plane. This is also the only model-independent method. In our framework, one can extrapolate to the complex energy plane by modifying the infinite-volume $G$ function in Eq. (8). Three two-body thresholds, i.e. $\pi \eta, K \bar{K}$ and $\pi \eta^{\prime}$, introduce $2^{3} \mathrm{RS}$ 's in the complex plane. The $G$ function for each channel has two RS's and the expression in Eq. (8) corresponds 
to the first RS. Its expression on the second RS takes the form

$$
G(s)_{I I}^{\mathrm{DR}}(s)=G(s)^{\mathrm{DR}}+i \frac{\sigma(s)}{8 \pi s},
$$

with $G(s)^{\mathrm{DR}}$ and $\sigma(s)$ defined in Eqs. (8) and (9), respectively 6 Changing from the first RS to the second one implies reversing the sign of the imaginary part of the $G$ function along the real $s$ axis above threshold.

We denote the physical/first RS by $(+,+,+)$, where the plus sign in each entry indicates that the $G$ function is evaluated in the physical RS at $\pi \eta, K \bar{K}$ and $\pi \eta^{\prime}$ thresholds, in order. The second, third, fourth and fifth RS are labeled as $(-,+,+),(-,-,+),(+,-,+)$ and $(-,-,-)$, respectively, with the minus sign indicating that the $G$ function for this channel is evaluated in its second RS, cf. Eq.(43). The same convention has also been used in Refs. [16,17], making the comparison between different approaches straightforward. In addition to the pole positions, we also calculate the residues for the three different channels, which characterize the couplings of the poles to the different channels.

Both for the LO and NLO cases, we find one relevant pole near the $K \bar{K}$ threshold, which is located either on the second or fourth sheet within $1 \sigma$ uncertainty. In fact, we find that the poles in the second sheet correspond to the parameter configurations that lead to the upper branch of phase shifts in Figs. 7 and 10, whereas the poles in the fourth sheet correspond to the parameters that give the lower branch of the phase shifts in Figs. 7 and 10, The relations between the transition of pole locations and the different behaviors of phase shifts are also noticed in Ref. [17. The explicit values of the pole positions, together with their residues, are given in Table 1. Notice that the central values of parameters of the LO and NLO fits lead to the $a_{0}(980)$ poles on the second and fourth sheets, respectively. At NLO, the poles around the $K \bar{K}$ threshold are quite similar to those at LO, though the masses of both the secondand fourth-sheet poles in the NLO amplitude are about $20 \mathrm{MeV}$ below the LO ones. We also note that the couplings to the $\pi \eta^{\prime}$ channel for the poles around the $K \bar{K}$ threshold in both LO and NLO cases are small, implying a marginal role of this channel when determining the $a_{0}(980)$ state. Let us note that no pole for the $a_{0}(980)$ in Table 1 lies in the unphysical RS that matches the physical RS above the $K \bar{K}$ threshold, so only the low-energy tail of the pole in the second RS is directly realized on the real energy axis below this threshold. One obvious difference between the LO and NLO amplitudes is that the latter contains a resonance pole located at around $1420 \mathrm{MeV}$ in the fifth RS, corresponding to the $a_{0}(1450)$, which is absent in the LO case. The $\pi \eta^{\prime}$ channel is found to be important for the heavy $a_{0}(1450)$ resonance, since the coupling to the $\pi \eta^{\prime}$ channel is even larger than to the coupling to $\pi \eta$ one for this resonance, as shown in Table 1.

Next we make a brief comparison with the pole content in Ref. [17]. Around the $K \bar{K}$ threshold region, one fourth-sheet pole is found, with mass $1177 \pm 27 \mathrm{MeV}$ and width $49 \pm 33 \mathrm{MeV}$. The error bars were obtained by averaging many different types of parametrizations in Ref. [17]. In our case the $a_{0}(980)$ pole can be either on the second or fourth sheet within $1 \sigma$ uncertainty. The pole content resulting from the global fits, by including both the lattice and experimental data, is summarized in Table 1, In order to make a more clear comparison with Ref. [17, we also give the pole contents from the LO fit by only including the 47 lattice energy levels. Again, the pole can be located either on the second or the fourth sheet within $1 \sigma$ uncertainty. The mass and width on the second sheet are $1170_{-26}^{+12} \mathrm{MeV}$ and $16_{-16}^{+34} \mathrm{MeV}$, respectively. The central value of the mass and width of the second sheet pole simply corresponds to taking the

\footnotetext{
${ }^{6}$ In the complex $s$ plane, $\sigma(s)$ has to be calculated such that $\operatorname{Im} \sigma(s)>0$.
} 
central value of the subtraction constant from this fit. For the mass and width of the fourthsheet pole, we take the median numbers as their central values. Then the mass and width of the pole on the fourth sheet are $1192_{-10}^{+11}$ and $12_{-12}^{+12} \mathrm{MeV}$. Similar rules are also applied to other numbers in Tables 11 and 2, As pointed out in Ref. [17] the lower half-plane of the second sheet is continuously connected to the upper half of the fourth sheet, which indicates that the nearby pole in the second or fourth sheet in fact represents quite similar physics. In Fig. 8 we further confirm this conclusion: The $S$ matrix exhibits continuous changes within uncertainties, though different sheets of poles are found.

Within uncertainties either a fourth-sheet virtual pole ranging from 971 to $978 \mathrm{MeV}$ near the $\pi \eta$ threshold or a third-sheet virtual pole ranging from 975 to $978 \mathrm{MeV}$ are found for the LO case, which confirms the result in Ref. [17, giving $964 \pm 62 \mathrm{MeV}$. However, at NLO we find only a prominent bump around $976 \mathrm{MeV}$ in the fourth sheet, instead of a pole. The fourth- or third-sheet virtual pole does not produce a prominent structure for the $\pi \eta$ scattering amplitude on the physical axis. Other poles that are far away from the $K \bar{K}$ threshold in the third sheet are also found in Ref. [17] and in our case. Since these poles are so far away from the energy region we are focusing on, we do not discuss them any further.

\begin{tabular}{cc|ccccc}
\hline \hline Resonance & RS & $\begin{array}{c}\text { Mass } \\
(\mathrm{MeV})\end{array}$ & $\begin{array}{c}\text { Width/2 } \\
(\mathrm{MeV})\end{array}$ & $\begin{array}{c}\mid \text { Residue }\left.\right|_{\pi \eta} ^{1 / 2} \\
(\mathrm{GeV})\end{array}$ & Ratios & \\
\hline LO & & & & & & \\
$a_{0}(980)$ & II & $1178_{-20}^{+4}$ & $3_{-3}^{+13}$ & $5.6_{-1.6}^{+0.1}$ & $1.23_{-0.01}^{+0.04}(K \bar{K} / \pi \eta)$ & $0.18_{-0.01}^{+0.02}\left(\pi \eta^{\prime} / \pi \eta\right)$ \\
$a_{0}(980)$ & IV & $1189_{-6}^{+15}$ & $4_{-4}^{+9}$ & $5.8_{-1.5}^{+0.3}$ & $1.21_{-0.03}^{+0.01}(K \bar{K} / \pi \eta)$ & $0.16_{-0.02}^{+0.01}\left(\pi \eta^{\prime} / \pi \eta\right)$ \\
\hline NLO & & & & & & \\
$a_{0}(980)$ & II & $1160_{-10}^{+14}$ & $2_{-2}^{+5}$ & $3.6_{-0.5}^{+0.9}$ & $1.29_{-0.03}^{+0.04}(K \bar{K} / \pi \eta)$ & $0.19_{-0.01}^{+0.00}\left(\pi \eta^{\prime} / \pi \eta\right)$ \\
$a_{0}(980)$ & IV & $1169_{-13}^{+26}$ & $4_{-4}^{+16}$ & $4.4_{-1.0}^{+1.4}$ & $1.25_{-0.05}^{+0.04}(K \bar{K} / \pi \eta)$ & $0.19_{-0.01}^{+0.01}\left(\pi \eta^{\prime} / \pi \eta\right)$ \\
$a_{0}(1450)$ & $\mathrm{V}$ & $1418_{-15}^{+13}$ & $54_{-18}^{+70}$ & $1.0_{-0.1}^{+0.8}$ & $2.9_{-0.9}^{+1.2}(K \bar{K} / \pi \eta)$ & $1.8_{-0.6}^{+0.3}\left(\pi \eta^{\prime} / \pi \eta\right)$ \\
\hline \hline
\end{tabular}

Table 1: Pole positions and the corresponding residues when the masses of pNGBs are taken at their lattice values in Eq. (36). The thresholds of $\pi \eta, K \bar{K}$ and $\pi \eta^{\prime}$ are 978.5, 1099, and 1321.1 MeV, respectively. We point out that there is only one pole around the $K \bar{K}$ threshold for each parameter configuration. Nevertheless, within $1 \sigma$ uncertainty different parameter configurations can either give a pole on the second sheet or the fourth sheet. See the text for details.

\section{Phase shifts, inelasticities and poles at the physical masses}

Since $U(3) \chi \mathrm{PT}$ is based on the chiral symmetry of QCD, it provides a useful framework to perform the chiral extrapolation from unphysically large pion masses to its physical value. Therefore, in this section, we give the predictions for the phase shifts, inelasticities, pole positions, and the residues for the $\pi \eta$ scattering by taking the physical masses for the $\pi, K, \eta$ and $\eta^{\prime}$ mesons.

As in the previous section, we present the results for the LO and NLO study separately. The LO predictions for the $\pi \eta$ phase shifts and inelasticities are shown in the left and right 
panels of Fig. 12, respectively. The corresponding predictions at NLO are given in Fig. 13. We observe very different results by comparing the two figures. Unlike the unphysical mass case, only one solution is found for LO. Although one solution is found for NLO around the $K \bar{K}$ threshold, two branches of phase shifts within $1 \sigma$ uncertainty appear at NLO in the energy region above around $1.4 \mathrm{GeV}$. To be more specific, we always observe a steep increase around the $K \bar{K}$ threshold for the LO $\pi \eta$ phase shifts, while the NLO phases continuously decrease above the $K \bar{K}$ threshold until the appearance of the $a_{0}(1450)$. In the energy region around 1.4 GeV, we find large uncertainties for the NLO phase shifts. However, the inelasticities turn out to be quite small in the same energy range. Then the situation here is similar to the discussions about large unphysical meson masses around the $1.2 \mathrm{GeV}$ region in Figs. 7 and 10. Due to the inclusion of the $a_{0}(1450)$, more complicated structures for the inelasticities appear at NLO than at LO. We also give the phase shifts and inelasticities from the $\pi \eta \rightarrow K \bar{K}$ scattering both at LO and NLO evaluated with physical masses in Fig. 14. We point out that the uncertainties given in Figs. 12, 13 and 14 should be taken with caution since only the statistical errors are included here; the systematic errors caused by the theoretical uncertainties and the chiral extrapolations are not considered. One possible theoretical uncertainty is given by using different pNGB decay constants in the scattering amplitudes. We make an exploratory study about this effect. The phase shifts and inelasticities with the replacement of $F_{\pi}$ by $F_{K}$ in the amplitudes involving kaons are shown as green dashed-dotted and dotted lines in Figs. 7 and 12, respectively. Quantitatively results similar to those from using a common $F_{\pi}$ in all the amplitudes are observed.
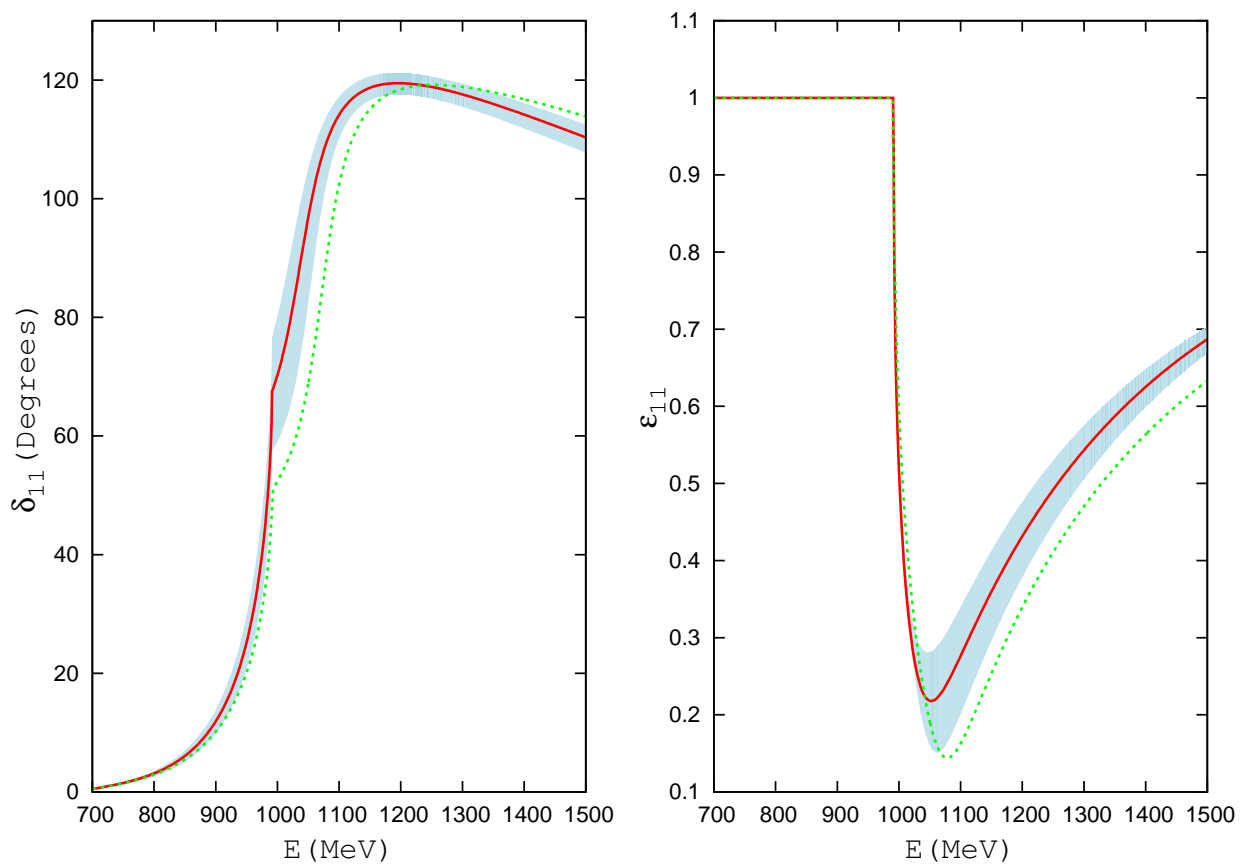

Figure 12: Phase shifts and inelasticities from $\pi \eta \rightarrow \pi \eta$ scattering with physical masses at leading order. The red solid lines correspond to the results from the best fit using a common pion decay constant in all the amplitudes. The shaded areas represent the statistical $1-\sigma$ uncertainties. The green dotted lines denote the results by distinguishing between $F_{\pi}$ and $F_{K}$ in the scattering amplitudes. See the text for details. 

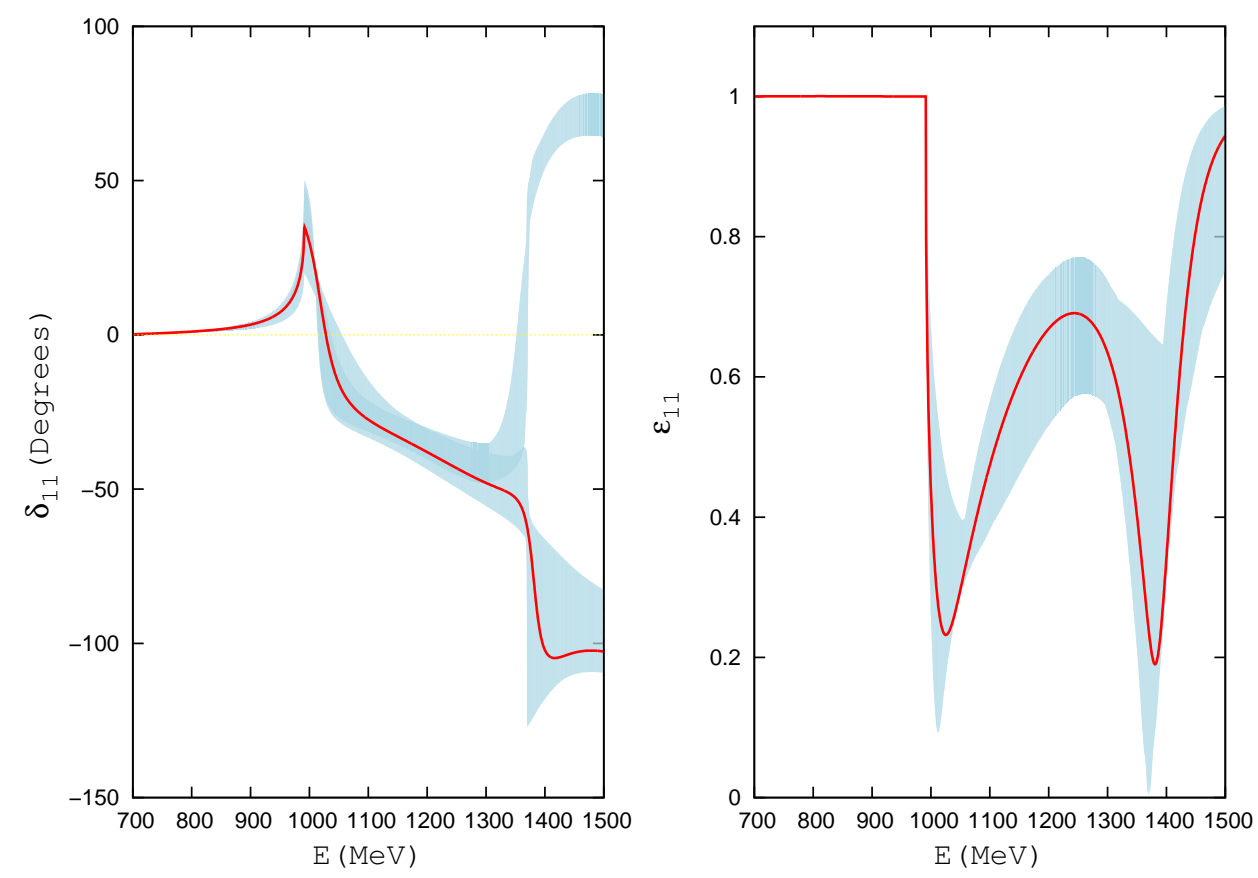

Figure 13: Phase shifts and inelasticities from $\pi \eta \rightarrow \pi \eta$ scattering with physical masses at next-to-leading order. The shaded areas represent the statistical 1- $\sigma$ uncertainties.
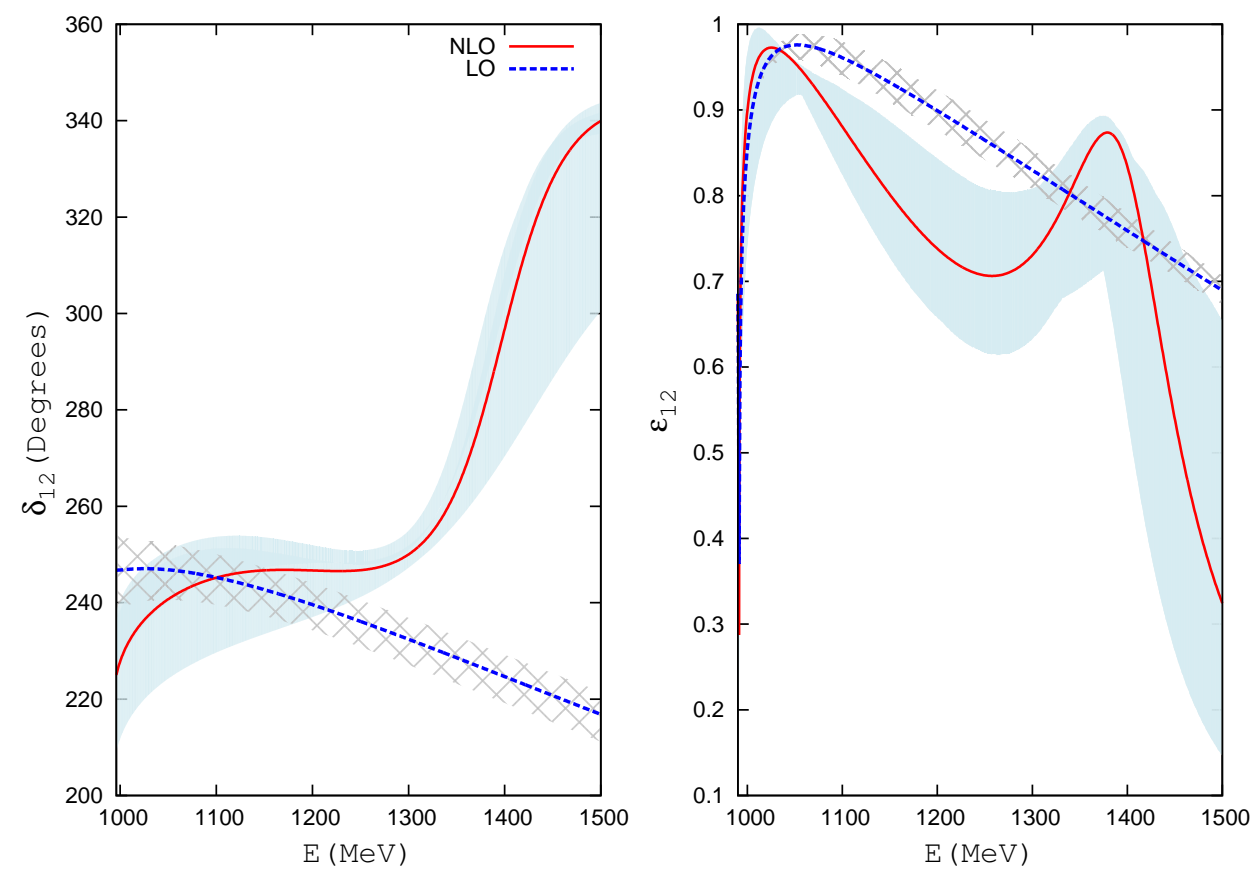

Figure 14: Phase shifts and inelasticities for the $\pi \eta \rightarrow K \bar{K}$ scattering with the physical masses for $\pi, K, \eta$ and $\eta^{\prime}$. For notations, see Fig. 9 . 
The relevant pole positions for the $a_{0}(980)$ and $a_{0}(1450)$ in the complex energy plane and the corresponding residues are given in Table 2, Only one second RS pole for the $a_{0}(980)$ is found in the LO case, while one pole located on the fourth RS is found in the NLO amplitude. The $a_{0}(980)$ poles in both cases are clearly above the $K \bar{K}$ threshold, and they are found to be barely coupled to the $\pi \eta^{\prime}$ channel. Neither of them lies in the RS that matches with the physical sheet in the energy interval between the $K \bar{K}$ and $\pi \eta^{\prime}$ thresholds. But, while for the LO case the pole in the second RS is directly accessible from the energy axis below this threshold, this is not the case for the hidden fourth RS pole in the NLO case. The most relevant pole for $a_{0}(1450)$ is located on the fifth RS since it lies above the $\pi \eta^{\prime}$ threshold. The coupling strength of the $a_{0}(1450)$ to the $\pi \eta^{\prime}$ channel is found to be similar to the $\pi \eta$ one, and therefore should be included when discussing this excited $a_{0}$ state. We mention that other redundant poles are also found in our unitarized amplitudes, such as a third-sheet pole with mass around $750 \mathrm{MeV}$ and width around $100 \mathrm{MeV}$. However, the redundant poles, which are usually located in the position that is not directly connected to the physical RS, do not show any visible effects on the physical axis. Therefore we refrain from discussing them any further.

Our current predictions for the $\pi \eta$ phase shifts are different from the recent study in Ref. [14. The reason for this is not difficult to understand. In that work, two specific pole positions for the $a_{0}(980)$ in the second and third RS's, and one pole for the $a_{0}(1450)$ in the third RS are taken as external input to determine the phase shifts. In other words, the phase shifts given in Ref. [14] are (at least partially) determined a priori by the imposed pole positions of the $a_{0}(980)$ and $a_{0}(1450)$. This is clearly different from our method, since the pole positions in Table 2 are not imposed beforehand. Instead, our pole content is determined once the phenomenological and lattice inputs are successfully reproduced. Indeed, we do not find any third RS pole for the $a_{0}(980)$ in our study, while in Ref. 14] this kind of pole is imposed to find the phase shifts. In our NLO study, we do not find any second RS pole and only one pole in the fourth RS is found. With different pole content embedded in the chiral amplitudes, it is not surprising to observe different solutions for the phase shifts. The phase shifts and inelasticities obtained here can provide important inputs for the dispersive study of processes involving $\pi \eta$ [68,69].

\begin{tabular}{cc|ccccc}
\hline \hline Resonance & RS & $\begin{array}{c}\text { Mass } \\
(\mathrm{MeV})\end{array}$ & $\begin{array}{c}\text { Width/2 } \\
(\mathrm{MeV})\end{array}$ & $\begin{array}{c}\mid \text { Residue }\left.\right|_{\pi \eta} ^{1 / 2} \\
(\mathrm{GeV})\end{array}$ & Ratios & \\
\hline LO & & & & & & \\
$a_{0}(980)$ & $\mathrm{II}$ & $1037_{-14}^{+17}$ & $44_{-9}^{+6}$ & $3.8_{-0.2}^{+0.3}$ & $1.43_{-0.03}^{+0.03}(K \bar{K} / \pi \eta)$ & $0.05_{-0.01}^{+0.01}\left(\pi \eta^{\prime} / \pi \eta\right)$ \\
\hline $\mathrm{NLO}$ & & & & & & \\
$a_{0}(980)$ & $\mathrm{IV}$ & $1019_{-8}^{+22}$ & $24_{-17}^{+57}$ & $2.8_{-0.6}^{+1.4}$ & $1.8_{-0.3}^{+0.1}(K \bar{K} / \pi \eta)$ & $0.01_{-0.01}^{+0.06}\left(\pi \eta^{\prime} / \pi \eta\right)$ \\
$a_{0}(1450)$ & $\mathrm{V}$ & $1397_{-27}^{+40}$ & $62_{-8}^{+79}$ & $1.7_{-0.4}^{+0.3}$ & $1.4_{-0.6}^{+2.4}(K \bar{K} / \pi \eta)$ & $0.9_{-0.2}^{+0.8}\left(\pi \eta^{\prime} / \pi \eta\right)$ \\
\hline \hline
\end{tabular}

Table 2: Pole positions and the corresponding residues when the masses of the pNGBs are fixed at their physical values given in Eq. (37). The thresholds of the $\pi \eta, K \bar{K}$, and $\pi \eta^{\prime}$ channels are 685.2, 991.2, and $1095 \mathrm{MeV}$, respectively. 


\section{Summary and conclusions}

In this work, we have analyzed very recent lattice finite-volume energy levels in the rest and moving frames for $\pi \eta$ scattering, together with the experimental data on a $\pi \eta$ event distribution and the $\gamma \gamma \rightarrow \pi \eta$ cross section. Three coupled channels, $\pi \eta, K \bar{K}$ and $\pi \eta^{\prime}$, are considered in our study. Both the leading and next-to-leading-order chiral amplitudes are used in the analyses. The simultaneous fits to the present lattice QCD finite-volume levels and the experimental data can not distinguish between the leading and next-to-leading-order scenarios, both of which lead to quite similar fit qualities.

However, somewhat different $\pi \eta$ scattering phase shifts are obtained for the leading and next-to-leading-order cases, when taking the heavy unphysical masses in Eq. (36). Two branches of solutions for the $\pi \eta$ phase shifts are found within uncertainties. Nevertheless, the two solutions of phase shifts in fact give similar dynamics, when combined with the inelasticities. The $\pi \eta \rightarrow K \bar{K}$ scattering phase shifts and inelasticities are also provided. A pole in either the second or the fourth Riemann sheet is found for the $a_{0}(980)$ within $1-\sigma$ uncertainty, when using the heavy unphysical masses for the $\pi, K, \eta$ and $\eta^{\prime}$. Our determinations for the pole of the $a_{0}(980)$ are compatible with those in Ref. [17] within uncertainties.

The most interesting predictions of this work are given in Sec. 5. The phase shifts and inelasticities of the $\pi \eta \rightarrow \pi \eta$ and $\pi \eta \rightarrow K \bar{K}$ scattering, pole positions and their residues are calculated by taking the physical masses for the $\pi, K, \eta$ and $\eta^{\prime}$. Within the statistical uncertainties, only one set of solutions of the $\pi \eta$ phase shifts is found for the leading-order case. Although one set of solutions of the $\pi \eta$ phase shifts is observed at next-to-leading order around the $K \bar{K}$ threshold, two branches of solutions are found above around $1.4 \mathrm{GeV}$. For the leading-order scenario, the physical $\pi \eta$ phase shifts clearly show a steep increase around the $K \bar{K}$ threshold. However, the phase shifts at next-to-leading order decrease continuously above this threshold until the appearance of the $a_{0}(1450)$ resonance. Though at next-to-leading order large uncertainties for the $\pi \eta$ phase shifts show up around $1.4 \mathrm{GeV}$, the inelasticities in the same region are quite small. The different behaviors of phase shifts are also reflected in the different pole contents. One pole slightly above the $K \bar{K}$ threshold is found in the second Riemann sheet for the leading-order amplitude (so that its low-energy tail directly influences the amplitudes on the energy axis below the $K \bar{K}$ threshold), while there is only one hidden fourth-sheet pole in the next-to-leading-order case for the $a_{0}(980)$. Due to the inclusion of the $a_{0}(1450)$ in the next-to-leading-order case, which is absent at leading order, the inelasticities from the two orders show different behaviors above around $1.1 \mathrm{GeV}$. The $\pi \eta^{\prime}$ channel is found to be rather weakly coupled to the $a_{0}(980)$ at both heavy unphysical and physical masses and hence plays a minor role for the determination of the $a_{0}(980)$ properties. The coupling strength of the $a_{0}(1450)$ to the $\pi \eta^{\prime}$ channel is nearly as large as the $\pi \eta$ one.

To summarize, global fits of similar quality including both experimental and lattice data are obtained, using unitarized chiral perturbation theory with two input chiral amplitudes, evaluated at leading and next-to-leading order. The leading-order amplitude gives a better description of the experimental $\pi \eta$ data evaluated at physical masses, but it gives slightly worse results for the lattice energy levels at $m_{\pi}=391 \mathrm{MeV}$. The situation for the next-to-leadingorder case is just the opposite. More importantly, the two different amplitudes obviously lead to different $\pi \eta$ phase shifts for the physical masses. Unlike the $\pi \eta$ experimental data which include the complicated $\pi \eta$ production mechanisms, the lattice energy levels are solely determined by the $\pi \eta$ scattering information. It is therefore important to have the finite-volume energy levels from the $\pi \eta$ scattering with lighter quark masses in order to discriminate between these two 
different solutions.

\section{Acknowledgements}

Finite-volume energy levels taken from Ref. [17] were provided by the Hadron Spectrum Collaboration - no endorsement on their part of the analysis presented in the current paper should be assumed. Z.H.G. would like to thank M. Albaladejo and J. J. Xie for communications, and M. Döring for many useful discussions. J.A.O. would like to expresses his gratitude to the HISKP for its kind hospitality during a research visit where part of this work was done. This work is carried out in the framework of the Sino-German Collaborative Research Center "Symmetries and the Emergence of Structure in QCD" (CRC 110) co-funded by the DFG and the NSFC. This work is also supported in part by the NSFC under Grants No. 11575052 and 11505038, the Natural Science Foundation of Hebei Province under Contract No. A2015205205, the MINECO (Spain) and ERDF (European Commission) Grant No. FPA2013-40483-P and by the Spanish Excellence Network on Hadronic Physics FIS2014-57026-REDT, grant by VolkswagenStiftung under Contract No. 86260. The work of U.G.M. was supported in part by The Chinese Academy of Sciences (CAS) President's International Fellowship Initiative (PIFI) Grant No. 2017VMA0025.

\section{References}

[1] C. Patrignani et al. [Particle Data Group], Chin. Phys. C 40, no. 10, 100001 (2016). doi:10.1088/1674-1137/40/10/100001

[2] J. A. Oller, Nucl. Phys. A727, 353 (2003) doi:10.1016/j.nuclphysa.2003.08.002 [hep$\mathrm{ph} / 0306031]$.

[3] A. Dobado, M. J. Herrero and T. N. Truong, Phys. Lett. B235, 134 (1990). doi:10.1016/0370-2693(90)90109-J.

[4] J. A. Oller and E. Oset, Nucl. Phys. A620, 438 (1997) Erratum: [Nucl. Phys. A652, 407 (1999)] doi:10.1016/S0375-9474(99)00427-3, 10.1016/S0375-9474(97)00160-7 [hep-ph/9702314].

[5] A. Dobado and J. R. Pelaez, Phys. Rev. D56, 3057 (1997) doi:10.1103/PhysRevD.56.3057 [hep-ph/9604416].

[6] J. A. Oller, E. Oset and J. R. Pelaez, Phys. Rev. D59, 074001 (1999) Erratum: [Phys. Rev. D60, 099906 (1999)] Erratum: [Phys. Rev. D75, 099903 (2007)] doi:10.1103/PhysRevD.59.074001, 10.1103/PhysRevD.60.099906, 10.1103/PhysRevD.75.099903 [hep-ph/9804209].

[7] J. A. Oller and E. Oset, Phys. Rev. D60, 074023 (1999) doi:10.1103/PhysRevD.60.074023 [hep-ph/9809337].

[8] J. R. Pelaez, Phys. Rept. 658, 1 (2016) doi:10.1016/j.physrep.2016.09.001 [arXiv:1510.00653 [hep-ph]].

[9] S. Descotes-Genon and B. Moussallam, Eur. Phys. J. C48, 553 (2006) doi:10.1140/epjc/s10052-006-0036-2 [hep-ph/0607133]. 
[10] H. Q. Zheng, Z. Y. Zhou, G. Y. Qin, Z. Xiao, J. J. Wang and N. Wu, Nucl. Phys. A733, 235 (2004) doi:10.1016/j.nuclphysa.2003.12.021 [hep-ph/0310293].

[11] J. R. Pelaez and A. Rodas, Phys. Rev. D93, no. 7, 074025 (2016) doi:10.1103/PhysRevD.93.074025 [arXiv:1602.08404 [hep-ph]].

[12] R. Garcia-Martin, R. Kaminski, J. R. Pelaez and J. Ruiz de Elvira, Phys. Rev. Lett. 107, 072001 (2011) doi:10.1103/PhysRevLett.107.072001 [arXiv:1107.1635 [hep-ph]].

[13] M. Albaladejo and J. A. Oller, Phys. Rev. Lett. 101, 252002 (2008) doi:10.1103/PhysRevLett.101.252002 [arXiv:0801.4929 [hep-ph]].

[14] M. Albaladejo and B. Moussallam, Eur. Phys. J. C75, no. 10, 488 (2015) doi:10.1140/epjc/s10052-015-3715-z [arXiv:1507.04526 [hep-ph]].

[15] T. Wolkanowski, F. Giacosa and D. H. Rischke, Phys. Rev. D93, no. 1, 014002 (2016) doi:10.1103/PhysRevD.93.014002 [arXiv:1508.00372 [hep-ph]].

[16] Z. H. Guo and J. A. Oller, Phys. Rev. D84, 034005 (2011) doi:10.1103/PhysRevD.84.034005 [arXiv:1104.2849 [hep-ph]].

[17] J. J. Dudek et al. [Hadron Spectrum Collaboration], Phys. Rev. D93, no. 9, 094506 (2016) doi:10.1103/PhysRevD.93.094506 [arXiv:1602.05122 [hep-ph]].

[18] M. Lüscher, Nucl. Phys. B354, 531 (1991). doi:10.1016/0550-3213(91)90366-6.

[19] M. Lage, U.-G. Meißner and A. Rusetsky, Phys. Lett. B681, 439 (2009) doi:10.1016/j.physletb.2009.10.055 [arXiv:0905.0069 [hep-lat]];

V. Bernard, M. Lage, U.-G. Meißner and A. Rusetsky, JHEP 1101, 019 (2011) doi:10.1007/JHEP01(2011)019 [arXiv:1010.6018 [hep-lat]].

[20] J. J. Dudek et al. [Hadron Spectrum Collaboration], Phys. Rev. Lett. 113, no. 18, 182001 (2014) doi:10.1103/PhysRevLett.113.182001 [arXiv:1406.4158 [hep-ph]];

D. J. Wilson, J. J. Dudek, R. G. Edwards and C. E. Thomas, Phys. Rev. D91, no. 5, 054008 (2015) doi:10.1103/PhysRevD.91.054008 [arXiv:1411.2004 [hep-ph]];

D. J. Wilson, R. A. Briceno, J. J. Dudek, R. G. Edwards and C. E. Thomas, Phys. Rev. D92, no. 9, 094502 (2015) doi:10.1103/PhysRevD.92.094502 [arXiv:1507.02599 [hep-ph]];

D. R. Bolton, R. A. Briceno and D. J. Wilson, Phys. Lett. B757, 50 (2016) doi:10.1016/j.physletb.2016.03.043 [arXiv:1507.07928 [hep-ph]];

R. A. Briceno, J. J. Dudek, R. G. Edwards and D. J. Wilson, Phys. Rev. Lett. 118, no. 2, 022002 (2017) doi:10.1103/PhysRevLett.118.022002. [arXiv:1607.05900 [hep-ph]];

G. Moir, M. Peardon, S. M. Ryan, C. E. Thomas and D. J. Wilson, JHEP 1610, 011 (2016) doi:10.1007/JHEP10(2016)011. [arXiv:1607.07093 [hep-lat]].

[21] C. B. Lang, L. Leskovec, D. Mohler and S. Prelovsek, Phys. Rev. D86, 054508 (2012) doi:10.1103/PhysRevD.86.054508 [arXiv:1207.3204 [hep-lat]];

D. Mohler, S. Prelovsek and R. M. Woloshyn, Phys. Rev. D87, no. 3, 034501 (2013) doi:10.1103/PhysRevD.87.034501 [arXiv:1208.4059 [hep-lat]]; 
S. Prelovsek and L. Leskovec, Phys. Rev. Lett. 111, 192001 (2013) doi:10.1103/PhysRevLett.111.192001 [arXiv:1307.5172 [hep-lat]];

D. Mohler, C. B. Lang, L. Leskovec, S. Prelovsek and R. M. Woloshyn, Phys. Rev. Lett. 111, no. 22, 222001 (2013) doi:10.1103/PhysRevLett.111.222001 [arXiv:1308.3175 [heplat]];

C. B. Lang, L. Leskovec, D. Mohler, S. Prelovsek and R. M. Woloshyn, Phys. Rev. D90, no. 3, 034510 (2014) doi:10.1103/PhysRevD.90.034510 [arXiv:1403.8103 [hep-lat]];

[22] D. Agadjanov, M. Döring, M. Mai, U.-G. Meißner and A. Rusetsky, JHEP 1606, 043 (2016) doi:10.1007/JHEP06(2016)043 [arXiv:1603.07205 [hep-lat]].

[23] J. A. Oller and U.-G. Meißner, Phys. Lett. B500, 263 (2001) doi:10.1016/S03702693(01)00078-8 [hep-ph/0011146].

[24] V. Bernard, M. Lage, U.-G. Meißner and A. Rusetsky, JHEP 1101, 019 (2011) doi:10.1007/JHEP01(2011)019 [arXiv:1010.6018 [hep-lat]].

[25] M. Döring, U.-G. Meißner, E. Oset and A. Rusetsky, Eur. Phys. J. A47, 139 (2011) doi:10.1140/epja/i2011-11139-7 [arXiv:1107.3988 [hep-lat]].

[26] M. Döring, U.-G. Meißner, E. Oset and A. Rusetsky, Eur. Phys. J. A48, 114 (2012) doi:10.1140/epja/i2012-12114-6 [arXiv:1205.4838 [hep-lat]].

[27] M. Döring and U.-G. Meißner, JHEP 1201, 009 (2012) doi:10.1007/JHEP01(2012)009 [arXiv:1111.0616 [hep-lat]].

[28] B. Hu, R. Molina, M. Döring and A. Alexandru, Phys. Rev. Lett. 117, no. 12, 122001 (2016) doi:10.1103/PhysRevLett.117.122001 [arXiv:1605.04823 [hep-lat]].

[29] T. A. Armstrong et al. [WA76 and Athens-Bari-Birmingham-CERN-College de France Collaborations], Z. Phys. C 52, 389 (1991). doi:10.1007/BF01559432.

[30] S. Uehara et al. [Belle Collaboration], Phys. Rev. D80, 032001 (2009) doi:10.1103/PhysRevD.80.032001 [arXiv:0906.1464 [hep-ex]].

[31] J. Gasser and H. Leutwyler, Nucl. Phys. B250, 465 (1985). doi:10.1016/05503213(85)90492-4.

[32] P. Herrera-Siklody, J. I. Latorre, P. Pascual and J. Taron, Nucl. Phys. B497, 345 (1997) doi:10.1016/S0550-3213(97)00260-5 [hep-ph/9610549].

[33] R. Kaiser and H. Leutwyler, Eur. Phys. J. C17, 623 (2000) doi:10.1007/s100520000499 [hep-ph/0007101].

[34] P. Di Vecchia and G. Veneziano, Nucl. Phys. B171, 253 (1980). doi:10.1016/05503213(80)90370-3;

C. Rosenzweig, J. Schechter and C. G. Trahern, Phys. Rev. D21, 3388 (1980). doi:10.1103/PhysRevD.21.3388;

E. Witten, Annals Phys. 128, 363 (1980). doi:10.1016/0003-4916(80)90325-5. 
[35] X. K. Guo, Z. H. Guo, J. A. Oller and J. J. Sanz-Cillero, JHEP 1506, 175 (2015) doi:10.1007/JHEP06(2015)175 [arXiv:1503.02248 [hep-ph]].

[36] M. Jamin, J. A. Oller and A. Pich, Nucl. Phys. B 587, 331 (2000) doi:10.1016/S05503213(00)00479-X [hep-ph/0006045].

[37] G. Ecker, J. Gasser, A. Pich and E. de Rafael, Nucl. Phys. B321, 311 (1989). doi:10.1016/0550-3213(89)90346-5.

[38] V. Bernard, N. Kaiser and U.-G. Meißner, Nucl. Phys. B364, 283 (1991). doi:10.1016/0550-3213(91)90586-M.

[39] J. A. Oller, Phys. Lett. B 477, 187 (2000) doi:10.1016/S0370-2693(00)00185-4 [hep$\mathrm{ph} / 9908493]$.

[40] C. D. Froggatt and J. L. Petersen, Nucl. Phys. B 129, 89 (1977). doi:10.1016/05503213(77)90021-9;

J. R. Batley et al. [NA48/2 Collaboration], Eur. Phys. J. C 54, 411 (2008). doi:10.1140/epjc/s10052-008-0547-0;

W. Ochs, PhD Thesis, University of Munich , 1974;

B. Hyams et al., Nucl. Phys. B 64, 134 (1973). doi:10.1016/0550-3213(73)90618-4;

P. Estabrooks et al., AIP Conf. Proc. 13, 37 (1973). doi:10.1063/1.2947126;

G. Grayer, et al., AIP Conf. Proc. 8, 5 (1972). doi:10.1063/1.2948709;

S. J. Lindenbaum and R. S. Longacre, Phys. Lett. B 274, 492 (1992). doi:10.1016/03702693(92)92022-9;

R. Kaminski, L. Lesniak and K. Rybicki, Z. Phys. C 74, 79 (1997) doi:10.1007/s002880050372 [hep-ph/9606362];

D. H. Cohen, D. S. Ayres, R. Diebold, S. L. Kramer, A. J. Pawlicki and A. B. Wicklund, Phys. Rev. D 22, 2595 (1980). doi:10.1103/PhysRevD.22.2595;

A. Etkin et al., Phys. Rev. D 25, 1786 (1982). doi:10.1103/PhysRevD.25.1786.

[41] S. D. Protopopescu et al., Phys. Rev. D 7, 1279 (1973). doi:10.1103/PhysRevD.7.1279;

P. Estabrooks and A. D. Martin, Nucl. Phys. B 79, 301 (1974). doi:10.1016/05503213(74)90488-X.

[42] W. Hoogland et al., Nucl. Phys. B 126, 109 (1977). doi:10.1016/0550-3213(77)90154-7;

M. J. Losty et al., Nucl. Phys. B 69 (1974) 185. doi:10.1016/0550-3213(74)90131-X.

[43] R. Mercer et al., Nucl. Phys. B 32, 381 (1971). doi:10.1016/0550-3213(71)90483-4;

P. Estabrooks, R. K. Carnegie, A. D. Martin, W. M. Dunwoodie, T. A. Lasinski and

D. W. G. S. Leith, Nucl. Phys. B 133, 490 (1978). doi:10.1016/0550-3213(78)90238-9;

H. H. Bingham et al., Nucl. Phys. B 41, 1 (1972). doi:10.1016/0550-3213(72)90419-1;

D. Aston et al., Nucl. Phys. B 296, 493 (1988). doi:10.1016/0550-3213(88)90028-4;

A. M. Bakker et al., Nucl. Phys. B 24, 211 (1970). doi:10.1016/0550-3213(70)90064-7;

Y. Cho et al., Phys. Lett. B 32, 409 (1970). doi:10.1016/0370-2693(70)90510-1. 
[44] U.-G. Meißner and J. A. Oller, Nucl. Phys. A679, 671 (2001) doi:10.1016/S03759474(00)00367-5 [hep-ph/0005253].

[45] Z. H. Guo, J. A. Oller and J. Ruiz de Elvira, Phys. Rev. D86, 054006 (2012) doi:10.1103/PhysRevD.86.054006 [arXiv:1206.4163 [hep-ph]].

[46] P. F. Bedaque, I. Sato and A. Walker-Loud, Phys. Rev. D73, 074501 (2006) doi:10.1103/PhysRevD.73.074501 [hep-lat/0601033].

[47] M. Albaladejo, J. A. Oller, E. Oset, G. Rios and L. Roca, JHEP 1208, 071 (2012) doi:10.1007/JHEP08(2012)071 [arXiv:1205.3582 [hep-lat]].

[48] M. Döring, M. Mai and U.-G. Meißner, Phys. Lett. B722, 185 (2013) doi:10.1016/j.physletb.2013.04.016 [arXiv:1302.4065 [hep-lat]].

[49] J. J. Xie and E. Oset, Eur. Phys. J. A48, 146 (2012) doi:10.1140/epja/i2012-12146-x [arXiv:1201.0149 [hep-ph]].

[50] H. X. Chen and E. Oset, Phys. Rev. D87, no. 1, 016014 (2013) doi:10.1103/PhysRevD.87.016014 [arXiv:1202.2787 [hep-lat]].

[51] D. Zhou, E. L. Cui, H. X. Chen, L. S. Geng and L. H. Zhu, Phys. Rev. D91, no. 9, 094505 (2015) doi:10.1103/PhysRevD.91.094505 [arXiv:1409.0178 [hep-lat]].

[52] L. S. Geng, X. L. Ren, Y. Zhou, H. X. Chen and E. Oset, Phys. Rev. D92, no. 1, 014029 (2015) doi:10.1103/PhysRevD.92.014029 [arXiv:1503.06633 [hep-ph]].

[53] M. Albaladejo, J. Nieves and P. Fernandez-Soler, Eur. Phys. J. C 76, no. 10, 573 (2016) doi:10.1140/epjc/s10052-016-4427-8 [arXiv:1606.03008 [hep-ph]].

[54] J. X. Lu, X. L. Ren and L. S. Geng, Eur. Phys. J. C 77, no. 2, 94 (2017) doi:10.1140/epjc/s10052-017-4660-9 [arXiv:1607.06327 [hep-ph]].

[55] A. Martinez Torres, L. R. Dai, C. Koren, D. Jido and E. Oset, Phys. Rev. D85, 014027 (2012) doi:10.1103/PhysRevD.85.014027 [arXiv:1109.0396 [hep-lat]].

[56] M. Göckeler, R. Horsley, M. Lage, U.-G. Meißner, P. E. L. Rakow, A. Rusetsky, G. Schierholz and J. M. Zanotti, Phys. Rev. D 86, 094513 (2012) [arXiv:1206.4141 [hep-lat]].

[57] L. Roca and E. Oset, Phys. Rev. D 85, 054507 (2012) doi:10.1103/PhysRevD.85.054507 [arXiv:1201.0438 [hep-lat]].

[58] J. J. Dudek, R. G. Edwards, B. Joo, M. J. Peardon, D. G. Richards and C. E. Thomas, Phys. Rev. D 83, 111502 (2011) doi:10.1103/PhysRevD.83.111502 [arXiv:1102.4299 [heplat]].

[59] J. J. Dudek et al. [Hadron Spectrum Collaboration], Phys. Rev. D 88, no. 9, 094505 (2013) doi:10.1103/PhysRevD.88.094505 [arXiv:1309.2608 [hep-lat]].

[60] C. Michael et al. [ETM Collaboration], Phys. Rev. Lett. 111, no. 18, 181602 (2013) doi:10.1103/PhysRevLett.111.181602 [arXiv:1310.1207 [hep-lat]];

K. Ottnad et al. [ETM Collaboration], Nucl. Phys. B896 (2015) 470 doi:10.1016/j.nuclphysb.2015.05.001 [arXiv:1501.02645 [hep-lat]]. 
[61] C. Davies and P. Lepage, AIP Conf. Proc. 717, 615 (2004) doi:10.1063/1.1799771 [hep$\mathrm{ph} / 0311041]$.

[62] C. T. H. Davies et al. [HPQCD and UKQCD and MILC and Fermilab Lattice Collaborations], Phys. Rev. Lett. 92, 022001 (2004) doi:10.1103/PhysRevLett.92.022001 [heplat/0304004].

[63] Y. Aoki et al. [RBC and UKQCD Collaborations], Phys. Rev. D83, 074508 (2011) doi:10.1103/PhysRevD.83.074508 [arXiv:1011.0892 [hep-lat]].

[64] R. Arthur et al. [RBC and UKQCD Collaborations], Phys. Rev. D87, 094514 (2013) doi:10.1103/PhysRevD.87.094514 [arXiv:1208.4412 [hep-lat]].

[65] Z. H. Guo and J. J. Sanz-Cillero, Phys. Rev. D89, no. 9, 094024 (2014) doi:10.1103/PhysRevD.89.094024 [arXiv:1403.0855 [hep-ph]].

[66] J. A. Oller and E. Oset, Nucl. Phys. A629, 739 (1998) doi:10.1016/S0375-9474(97)00649-0 [hep-ph/9706487].

[67] J. A. Oller, Nucl. Phys. A714, 161 (2003) doi:10.1016/S0375-9474(02)01360-X [hep$\mathrm{ph} / 0205121]$.

[68] R. Escribano, S. Gonzalez-Solis and P. Roig, Phys. Rev. D 94, no. 3, 034008 (2016) doi:10.1103/PhysRevD.94.034008 [arXiv:1601.03989 [hep-ph]].

[69] M. Albaladejo, J. T. Daub, C. Hanhart, B. Kubis and B. Moussallam, arXiv:1611.03502 [hep-ph]. 